Wissenschaftlicher Fortschritt in den Wirtschaftswissenschaften: Einige Bemerkungen

Gebhard Kirchgässner

April 2011 Discussion Paper no. 2011-16 


$\begin{array}{ll}\text { Editor: } & \text { Martina Flockerzi } \\ & \text { University of St. Gallen } \\ & \text { School of Economics and Political Science } \\ & \text { Department of Economics } \\ & \text { Varnbüelstrasse } 19 \\ & \text { CH-9000 St. Gallen } \\ & \text { Phone } \quad+41712242325 \\ & \text { Fax } \quad+41712243135 \\ & \text { Email seps@unisg.ch } \\ & \text { School of Economics and Political Science } \\ & \text { Department of Economics } \\ & \text { University of St. Gallen } \\ & \text { Varnbüelstrasse 19 } \\ \text { Publisher: } & \text { CH-9000 St. Gallen } \\ & \text { Phone +41 71 224 23 25 } \\ & \text { Fax } \quad+41712243135 \\ & \text { http://www.seps.unisg.ch }\end{array}$




\title{
Wissenschaftlicher Fortschritt in den Wirtschaftswissenschaften: Einige Bemerkungen ${ }^{1}$
}

\author{
Gebhard Kirchgässner²
}

Author's address:

Prof. Dr. Gebhard Kirchgässner

SIAW-HSG

Bodanstrasse 8

Phone +41712242340

Fax +41712242298

Email gebhard.kirchgaessner@unisg.ch

Website www.siaw.unisg.ch

\footnotetext{
${ }^{1}$ Vortrag im Rahmen des Internationalen Symposiums „Philosophie und Wirtschaftswissenschaft“ aus Anlass des 90. Geburtstags von Prof. Hans Albert, Klagenfurt, 3. - 5. Februar 2011. Überarbeitete schriftliche Fassung, März 2011.

2 University of St. Gallen, Swiss Institute for International Economics and Applied Economic Research, Leopoldina and CESifo.
} 


\begin{abstract}
First, the points of view of economists regarding falsificationism, scientific revolutions and scientific research programmes are discussed. Next, hardly debatable scientific progress regarding empirical economic research in recent decades is described. Then it is asked whether there have been scientific revolutions with respect to economic theory or the basic methodology of the economic approach. Taking this term seriously, there have been at best two revolutions since the time of Adam Smith. Today, economists share a common paradigm, which also builds the hard core of their scientific research programme. But while this hard core is hardly questioned, the safety belt is discussed the more. Nevertheless, most today's economic research can be considered as being 'normal science'. Even if this kind of research is not without problems, there is no reason to assess it as being of secondary value.
\end{abstract}

\title{
Keywords
}

Paradigm, normal science, methodology of scientific research programmes, empirical economic research.

\section{JEL Classification}

B10; B41 


\section{Einleitung}

[1] In wieweit es in den Wirtschaftswissenschaften Fortschritt gibt, ist ein altes Thema, welches letztlich im 19. Jahrhundert auch der Diskussion zwischen der Historischen Schule und den ,Österreichern“ zugrunde lag. Andererseits wird in der ökonomischen Literatur, soweit sie sich mit grundlegenden methodologischen Fragen auseinandersetzt, das Problem des wissenschaftlichen Fortschritts kaum erörtert; es scheint zu selbstverständlich zu sein, dass derartiger Fortschritt stattfindet. Man befasst sich dann z.B. mit der Rolle von Theorien oder der Empirie, gelegentlich wie z.B. in den ,Economics of Economics ${ }^{6}$ auch mit den Bedingungen, unter denen sich wissenschaftliche Forschung in den Wirtschaftswissenschaften vollzieht. ${ }^{1)}$ Dabei geht es in jüngerer Zeit vor allem um die Organisation des Wissenschaftsbetriebs und dabei insbesondere um Fragen der Publikationen und ihrer Bedeutung im Wissenschaftsprozess. ${ }^{2)}$ Dabei wird auch gefragt, wie sich die (akademische) Forschung in den Wirtschaftswissenschaften in Zukunft weiterentwickeln wird. ${ }^{3)}$ Dort, wo die Frage der Produktivität der Forscher erörtert wird, geht es fast ausschliesslich um Rankings, die sich aus der Zahl der Publikationen bzw. Zitierungen ergeben. ${ }^{4)}$ Fragen nach den Bedingungen für wissenschaftlichen Fortschritt werden dagegen eher selten erörtert, wenn z.B. (ökonomische) Wissenschaft als freier Markt für Ideen verstanden und die Zulassung von Pluralismus bezüglich der Methoden und Ansätze gefordert wird. ${ }^{5)}$

[2] In allerjüngster Zeit ist die Frage nach dem Fortschritt in den Wirtschaftswissenschaften sowie insbesondere der Volkswirtschaftslehre jedoch insofern wieder in die Diskussion gekommen, als im Gefolge der Wirtschaftskrise nicht nur die Theorie der Finanzmärkte, sondern insbesondere die makroökonomische Theorie in Misskredit geraten ist. ${ }^{6)}$ Während manche eine Rückkehr zur (keynesianischen) Theorie der siebziger Jahre verlangten, die in der praktischen Wirtschaftspolitik durch die massiven Konjunkturstützungsprogramme ja auch zumindest vorübergehend teilweise vollzogen wurde, ${ }^{7)}$ forderten in Deutschland eine ganze Reihe von Ökonomen in einem öffentlichen Apell eine Abkehr von der modernen, von den

1. Zur Economics of Economics siehe z.B. T. EGGERTSSON (1994), B.S. FREY (2000), T. COUPÉ (2004) sowie die Beiträge in German Economic Review 9 (2008, Heft 4).

2. Siehe z.B. T. Coupé (2004, S. 200ff.), B.S. Frey (2005) sowie M. Graber, A. LAunOv und K. WÄlde (2008).

3. Siehe z.B. B.S. FREY (2010).

4. Siehe z.B. O. FABel, M. HeIN und R. HOFMEISTER (2008) für die Betriebswirtschaftslehre bzw. die Definition der „forschungsstärksten Professoren“ im Handelsblatt Ranking der TOP-Fakultäten für Volkswirtschaftslehre, $\quad \mathrm{http}: / / \mathrm{www} \cdot h a n d e l s b l a t t . c o m / p o l i t i k / v w l-r a n k i n g / h a n d e l s b l a t t-r a n k i n g-v w l-2010$-werdeutschlands-top-oekonomen-sind;2537793 (11/02/11).

5. Siehe z.B. U. MÄKI (2000). Eine Ausnahme bildet R.E. BACKHOUSE (1997), der sich u.a. intensiv damit auseinandersetzt, wann man in der Wirtschaftswissenschaft von Fortschritt reden kann. (Siehe insbesondere S. 97ff.) Siehe auch J. SHEARMUR (1991),

6. Siehe hierzu z.B. D. ACEMOGLU (2009) sowie die entsprechenden Beiträge im Economist vom 16. Juni 2009 (http://www.economist.com/node/14031376, http://www.economist.com/node/14030288, http://www. economist.com/node/14030296 (11/02/11).

7. Siehe z.B. P. Krugman, How Did Economists Get It So Wrong, New York Times vom 6. September 2009. (http://www.nytimes.com/2009/09/06/magazine/06Economic-t.html (11/02/11).) 
angelsächsischen Ländern dominierten empirisch-quantitativen Ausrichtung der Volkswirtschaftslehre und eine Rückkehr zur deutschen Ordnungsökonomik, die im Gegensatz zur modernen Ökonomik weitgehend ohne Mathematik auskam. ${ }^{8)}$ In diesem Zusammenhang wurde zwar nicht grundsätzlich verneint, dass es Fortschritt im Bereich der Wirtschaftswissenschaften geben kann, aber zumindest wurde die Entwicklung der letzten Jahrzehnte eher als Rückdenn als Fortschritt betrachtet. ${ }^{9)}$

[3] Fragt man die Wissenschaftstheorie, wann man von wissenschaftlichem Fortschritt reden kann, wird man zwangsläufig auf die Diskussionen der sechziger und siebziger Jahre verwiesen, deren Exponenten Karl R. Popper, Thomas Kuhn, ImRe LaKatos und Paul K. FEYERABEND waren. ${ }^{10)}$ Diese Diskussion litt u.a. darunter, dass analytische und historischdeskriptive Elemente miteinander vermischt wurden. Während es bei K.R. POPPER im Wesentlichen darum geht, wann eine Theorie als besser als eine Konkurrentin ausgewiesen werden kann, geht es TH. KuHN (1962) vor allem um eine Analyse der Prozesse, in denen eine Theorie durch eine andere abgelöst wird (wobei es beiden um eine Rückweisung der ,Kübeltheorie' wissenschaftlichen Fortschritts geht). Als Ergebnis dieser Diskussionen kann man u.a. festhalten, dass man nie sicher sein kann, ob die Theorie, die man für die bessere hält, auch tatsächlich überlegen ist; alle Versuche zu zeigen, dass eine Theorie näher an der Wahrheit ist als eine Konkurrentin, müssen wohl als gescheitert angesehen werden. Selbst wenn alle Kriterien erfüllt sind, gemäss denen nach K.R. POPPER (1963, S. 240ff.) eine Theorie als besser als ihre Konkurrentin ausgewiesen werden soll, kann nicht ausgeschlossen werden, dass neue Tests vorgeschlagen werden, bei denen die ,alte' Theorie besser abschneidet. Nicht umsonst spricht auch K.R. POPPER (1972, S. 70ff.) zwar von Wahrheitsähnlichkeit als Ziel der Theoriebildung, aber er betont auch, dass es keine Metrik gibt, mit welcher man die Wahrheitsähnlichkeit einer Theorie abschätzen könne, und „dass es nie möglich ist, eindeutig zu beweisen, dass eine empirisch-wissenschaftliche Theorie falsch ist"“ (1983, S. XVI).

[4] Wir befinden uns damit bei der Diskussion wissenschaftlichen Fortschritts in einer ähnlichen Situation wie beim Induktionsproblem: Obwohl das Problem theoretisch nicht gelöst ist und aller Voraussicht auch gar nie gelöst werden kann, führen wir nicht nur permanent praktische Induktionsschlüsse erfolgreich durch, sondern wir sind auch alle davon überzeugt, dass es wissenschaftlichen Fortschritt gibt, und wir urteilen darüber, welche Theorie wir für besser halten, auch wenn der Konsens darüber im Allgemeinen in den Sozialwissenschaften geringer als in den Naturwissenschaften ist. Schliesslich kann man kaum ernsthaft bezweifeln, dass uns heute dank der Wissenschaft Möglichkeiten offenstehen, die frühere Generationen

8. Diese Debatte wurde vorwiegend in allgemeinen Zeitungen sowie im Internet ausgetragen. Siehe z.B. G. SCHWARZ, Die trostlose Wirtschaftswissenschaft, Neue Zürcher Zeitung Nr. 84 vom 11./12. April 2009, S. 19, V. VANBERG, Die Ökonomik ist keine zweite Physik, Frankfurter Allgemeine Zeitung (FAZ-NET) vom 13. April 2009, http://www.faz.net/s/RubB8DFB31915A443D98590B0D538FC0BEC/Doc $\sim$ EF85E9DC12 CF9465697F08B975B0D79C7 ATpl Ecommon Scontent.html (11/02/11), sowie der in eine ähnliche Richtung zielende Aufruf der 83 Professoren „Rettet die Wirtschaftspolitik an den Universitäten“" vom 5. Mai 2009 (Frankfurter Allgemeine Zeitung (FAZ-NET)) ,http:/www.faz.net/s/ RubB8DFB31915A443 D98590B0D538FC0BEC/Doc EA1E6687105BC44399 168BC77ADE64F8A ATpl Ecommon Scontent.html (01.02.11).

9. Zu dieser Diskussion siehe G. KIRCHGÄSSNER (2009).

10. Siehe hierzu z.B. die Beiträge in I. LAKATOs und A. Musgrave (1974). 
nicht zur Verfügung hatten. Aus rein instrumentellen Überlegungen heraus kommen wir zur Überzeugung, dass der Stand der Wissenschaft heute insgesamt weiter ist, als er früher war, auch wenn wir im Einzelnen nicht sagen können, ob eine bestimmte Theorie wirklich besser als eine andere ist.

[5] Diese instrumentelle Sicht der Wissenschaft wurde von M. FRIEDMAN (1953) auf die Ökonomie übertragen. Diese Arbeit löste eine bis heute andauernde Diskussion aus. ${ }^{11)}$ Aber selbst wenn man diese Perspektive akzeptiert, bringt dies für die Diskussion der letzten Jahre über die makroökonomische Theorie nichts, da mit dem Verweis darauf, dass man für die Bewältigung der Krise zur alten, auf der keynesianischen Theorie basierenden Politik zurückkehren müsse, ja insbesondere die instrumentelle Eignung der neueren Ansätze in Frage gestellt wurde. Ähnliche Entwicklungen gibt es auch in anderen Bereichen. So wurde in den letzten Jahrzehnten die Theorie einer strategischen Handelspolitik entwickelt, die jedoch nicht nur in der Praxis keine Anwendung findet, sondern auch von Ökonomen als mehr oder weniger untauglich für eine praktische Umsetzung betrachtet wird. ${ }^{12)}$ Für die Wirtschaftspolitik vertrauen Wissenschaftler wie Politiker - möglicherweise durchaus zu Recht - nach wie vor eher auf die klassische Theorie von DAVID RICARDO als auf die Entwicklungen der modernen Aussenhandelstheorie. ${ }^{13)}$

[6] Für die Diskussion über wissenschaftlichen Fortschritt in den Wirtschaftswissenschaften sind aus der Diskussion der sechziger und siebziger Jahre jedoch vor allem zwei andere Punkte relevant, die auch entsprechend aufgegriffen wurden: (i) Gibt es in den Wirtschaftswissenschaften Paradigmen und wissenschaftliche Revolutionen (im Sinne von TH.S. KUHN (1962)) und welche Rolle spielt in diesem Zusammenhang die Normalwissenschaft, und (ii) gibt es (im Sinne von I. LAKATOS (1974)) wissenschaftliche Forschungsprogramme. Gerade dieses Konzept hat in den Wirtschaftswissenschaften weite Beachtung gefunden. Dies ist nicht überraschend; es resultiert aber zumindest zum Teil aus einer eher merkwürdigen Auffassung des Popperschen Falsifikationismus (an der er selbst nicht ganz unschuldig ist), die mit dem tatsächlichen Wissenschaftsbetrieb nicht vereinbar ist, und der ,Absolution', welche die Konzeption von I. LAKATOS (1974) dem Wissenschaftler für sein Verhalten zu erteilen vorgibt.

[7] Dabei gibt es im empirisch methodischen Bereich seit dem 2. Weltkrieg Fortschritte, die als solche kaum bestritten werden dürften, auch wenn, wie bereits erwähnt wurde, gelegentlich der gesamte empirische Ansatz für die Volkswirtschaftslehre als verfehlt angesehen wird. Dieser Fortschritt hat mit der Entwicklung leistungsfähiger Rechner, der Erfassung von immer mehr Daten und der Entwicklung darauf besser abgestimmter statistisch-ökonometrischer Verfahren zu tun. Dies bedeutet freilich nicht notwendigerweise, dass damit auch die ökonomischen Theorien, die mit solchen Verfahren getestet werden sollen, ,besser' geworden sind.

[8] Im Folgenden soll zunächst auf die Positionen verschiedener Ökonomen im Rahmen der Debatte um Falsifikationismus, wissenschaftliche Revolutionen und wissenschaftliche For-

11. Siehe hierzu z.B. M. BLAUG (1980, S. 91 ff.) sowie die Beiträge in U. MÄKI (2009).

12. Siehe hierzu R.E. BALDWIN (1992).

13. Siehe hierzu z.B. P. KRUGMAN (1987). 
schungsprogramme eingegangen werden (Abschnitt 2). Danach wird der kaum bestreitbare Fortschritt dargestellt, der sich in den letzten Jahrzehnen im methodisch-empirischen Bereich vollzogen hat und der zu einer neuen, wenn auch aller Voraussicht nach nur vorübergehenden Version des ökonomischen Imperialismus in anderen Sozialwissenschaften geführt hat $(A b$ schnitt 3). Im Anschluss daran wird gefragt, ob es neben diesem Fortschritt, der sich vergleichsweise kontinuierlich vollzieht, auch Revolutionen gegeben hat, die sich insbesondere im Bereich der Theorie (bzw. der grundlegenden Methodologie) vollzogen haben (Abschnitt 4). Abschnitt 5 befasst sich mit dem Paradigma, welches hinter dem ökonomischen Ansatz steht, und Abschnitt 6 mit dem auf diesem Paradigma aufbauenden Forschungsprogramm Wirtschaftswissenschaften und der Kritik daran, die insbesondere in jüngerer Zeit daran geübt wird. Bei all dem soll es nicht um die Frage gehen, wie die Konzeptionen von TH.S. KuHN und I. LAKATOS insgesamt als Erklärungsansätze für die Entwicklung der Wirtschaftswissenschaften zu beurteilen (bzw. ob sie zu verwerfen) sind, sondern lediglich darum, inwieweit einzelne Kernpunkte dieser Ansätze helfen können (oder auch nicht), bestimmte Einwicklungen innerhalb der Wirtschaftswissenschaften zu verstehen. ${ }^{14)}$ In Abschnitt 7 diskutieren wir schliesslich Probleme der Normalwissenschaft in den Wirtschaftswissenschaften, die heute ohne Zweifel den grössten Raum einnimmt. Auch wenn damit einige Probleme verbunden sind, besteht kein Anlass, diese Forschung in irgendeiner Weise abzuwerten.

\section{Die wissenschaftstheoretische Debatte um den Fortschritt in den Wissenschaften und die Wirtschaftswissenschaft}

[9] Sozialwissenschaftler und insbesondere Ökonomen haben zum Kritischen Rationalismus häufig eine ambivalente Einstellung. Zum einen leuchtet die Asymmetrie bezüglich Verifikation und Falsifikation von All- und Existenzaussagen unmittelbar ein. Zudem ist es heute zumindest bei den Wissenschaftlern, die empirisch arbeiten, üblich, zunächst ein Modell zu entwickeln und dieses dann ,empirisch zu überprüfen', d.h. die Parameter der entsprechenden Gleichungen mit Hilfe statistischer Verfahren zu schätzen und anschliessend statistische Tests durchzuführen. ${ }^{15)}$ Dieses Verfahren scheint demjenigen, welches der Kritische Rationalismus fordert, zumindest in etwa zu entsprechen. ${ }^{16)}$ Viele, vielleicht sogar die meisten Ökonomen bekennen sich zumindest verbal zum Kritischen Rationalismus, auch wenn sie sich eher selten explizit auf K.R. POPPER berufen. Dennoch ist er unter Ökonomen ausgesprochen populär. ${ }^{17}$ Viele vertreten dabei allerdings eher einen ,naiven' als einen ,raffinierten' Falsifikationismus, um die Terminologie von I. LAKATOS (1974, S. 113ff.) zu verwenden. Dabei gehen sie zumeist von der Annahme aus, dass sich Existenzaussagen endgültig verifizieren und Allaussa-

14. In wieweit der Ansatz von TH.S. KUHN (1991) sich überhaupt eignet, die Entwicklung der Wissenschaften zu beschreiben, ist auch für die Naturwissenschaften umstritten. Siehe hierzu die Kritik an diesem Ansatz durch G. ANDERSSON (1988) sowie die Besprechung dieser Arbeit durch H. ALBERT (1990). Weitere Beiträge dazu finden sich z.B. in Social Epistomology 17 (2003), Heft 2/3.

15. Zum Testen ökonomischer Theorien siehe J. KIM (1991) sowie CH.L. GILBERT (1991).

16. Zur Beurteilung der Ökonomie aus der Sicht des Kritischen Rationalismus siehe H. ALBERT (1963), aber auch H. AlBERT $(1978,1986)$. Zur Anwendungsproblematik des Kritischen Rationalismus in der Ökonomie siehe W. MEYER (1973).

17. Siehe hierzu auch B.J. CALDWELL (1991, S. 30f.). 
gen endgültig falsifizieren lassen, eine Auffassung, die sich bekanntlich nicht aufrecht erhalten lässt und worauf K.R. POPPER bereits 1935 hingewiesen hat. ${ }^{18)}$

[10] Andererseits hat wohl kaum ein Wissenschaftler ein Interesse daran, seine eigenen Hypothesen und Theorien zu widerlegen. Vielmehr versuchen Wissenschaftler üblicherweise genau das Gegenteil: Sie sammeln alles an Evidenz, was für ihre Theorien spricht, sie negieren häufig gegenteilige Evidenz und versuchen gelegentlich sogar, sie zu unterdrücken. Wenn es nicht mehr anders geht, ,erweitern' sie ihre Theorien, um sie mit zunächst gegen sie sprechenden Beobachtungen verträglich zu machen. Dies verringert in aller Regel den empirischen Gehalt. Solche Verhaltensweisen scheinen eher mit dem Ansatz von TH.S. KUHN (1962) vereinbar zu sein als mit den Ideen K.R. PopPERs. ${ }^{19)}$ Daher halten viele das Vorgehen im empirischen Bereich der ökonomischen Wissenschaften genauso wie die Arbeit in den anderen Realwissenschaften für unvereinbar mit dessen Ansatz. ${ }^{20)}$ Dies wirft die Frage nach der möglichen Bedeutung dieser Theorie auf: Welcher Stellenwert kann einer Theorie der Wissenschaft zukommen, die täglich vom tatsächlichen wissenschaftlichen Geschehen ,falsifiziert' wird?

[11] Vor diesem Hintergrund ist es verständlich, dass (nicht nur) Ökonomen fast dankbar waren für den Ansatz, den I. LAKATOS (1974) ihnen mit seiner Methodologie wissenschaftlicher Forschungsprogramme geliefert hat. ${ }^{21)}$ Jetzt war es nicht mehr gefordert, dass sie ihre eigenen Theorien zu widerlegen versuchen, sondern sie konnten sich auf den Kern des Programms zurück ziehen, der zwar nicht notwendigerweise als unwiderlegbar angesehen werden muss, der aber als solcher zumindest vorläufig akzeptiert werden darf, und Veränderungen ausserhalb dieses Bereichs sind intellektuell zulässig, da nur durch die Verteidigung einer Theorie gegen Angriffe ihr Gehalt wirklich voll erfasst werden kann; es kann problematisch sein, Theorien zu früh fallen zu lassen.

[12] Dies hat dazu geführt, dass - beginnend mit S. LATSIS (1972) - verschiedene Ökonomen begannen, die Methodologie der wissenschaftlichen Forschungsprogramme auf Frage-

18. Siehe hierzu auch K.R. POPPER (1983, S. XVI). Auf die Unmöglichkeit einer endgültigen Falsifikation einzelner theoretischer Hypothesen hatten zuvor bereits PIERRE DUHEM und später auch WILLARD VAN ORMAN QUINE hingewiesen, weshalb man diesbezüglich auch von der Duhem bzw. Duhem-Quine These spricht. Siehe hierzu z.B. D.A. Redman (1991, S. 38f.). M. Blaug (1976, S. 354) weist darauf hin, dass sich entsprechende Überlegungen bereits bei HENRI POINCARÉ finden.

19. Siehe hierzu auch D.W. HANDS (1985, S. 2.): „Unabhängig davon, wie sehr die ökonomische Profession auch immer den Falsifikationismus predigt, es ist der allgemeine Konsens derjenigen, die in jüngerer Zeit deren Vorgehensweise kommentiert haben, dass sie diesen kaum jemals praktizieren.“ Und M. BLAUG (1980, S. 111) schreibt: „Moderne Ökonomen predigen häufig der Falsifikationismus, wie wir gesehen haben, aber sie praktizieren ihn selten, die von ihren verwendete Wissenschaftstheorie kann passend als ,harmloser Falsifikationismus' beschrieben werden.“

20. Siehe z.B. D.M. Hausman (1989, S. 121f.) sowie H. Müller-GuefFroy (1985, S. 146), der die Auffassung vertritt, der Gegenstandsbereich der Nationalökonomie erzwinge „die Formulierung von Theorien hoher Allgemeinheit und damit von Theorien mit geringer Falsifizierbarkeit.“

21. Siehe hierzu auch D.W. HAND (1985, S. 2) sowie D.A. REDMAN (1993, S. 142ff.). M. BLAUG (1976, s. 353) sieht die Konzeption der wissenschaftlichen Forschungsprogramme als ,einen Kompromiss zischen Poppers ,aggressiver Methodologie“ und Kuhns ,defensiver Methodologie“". Auch I. LAKATOS (1974, S. XX) sieht seinen Ansatz als Kompromiss zwischen den Ansätzen von K.R. POPPER und TH.S. KUHN. 
stellungen bzw. Entwicklungen in den Wirtschaftswissenschaften anzuwenden. ${ }^{22)}$ Dies vollzog sich im Wesentlichen in den achtziger Jahren. Nach einer anfänglichen Euphorie nahm aber, wie R.E. BACKHOUSE (2004, S. 191ff.) beschreibt, des Skeptizismus unter den methodologisch interessierten Ökonomen zu, und heute scheint dieses Programm weitgehend diskreditiert zu sein, auch wenn er (S. 193) davor warnt, es zu schnell zu verabschieden, und dafür auch einige beachtenswerte Gründe angibt. ${ }^{23}$ Während andere (wie z.B. D. MCCLOSKEY (1983)) damit das zugrunde liegende Programm von K.R. POPPER insgesamt verabschieden möchten, weist er darauf hin, dass man das Programm von I. LAKATOS (1974) nicht unbedingt in der Tradition von K.R. POPPER sehen muss, sondern dass man es auch in eine älteren Tradition, die bis auf GEORG WILHELM FRIEDRICH HEGEL zurückgeht, einordnen sowie vor dem Hintergrund seiner Wissenschaftstheorie der Mathematik sehen kann, wodurch es in einem sehr viel positiveren Licht erscheine. ${ }^{24)}$ Dessen ungeachtet wird in der ökonomischen Literatur heute nur noch vergleichsweise selten auf I. LAKATOS Bezug genommen. ${ }^{25)}$

[13] Die Zurückweisung des Kritischen Rationalismus durch viele Wissenschaftstheoretiker und methodologisch orientierte Ökonomen lässt zum einen die Frage aufkommen, weshalb K.R. POPPER, wie oben bereits ausgeführt wurde, unter den Ökonomen dennoch so populär ist, obwohl sie seinen Anweisungen nicht folgen bzw. dessen Konzeption durch die tägliche Wissenschaftspraxis widerlegt scheint. ${ }^{26)}$ Wichtiger aber dürfte die Frage sein, ob diese Konzeption durch diese Praxis tatsächlich widerlegt wird. Dieser Behauptung liegt - meines Erachtens - zumindest ein doppeltes Missverständnis zugrunde, wobei freilich K.R. POPPER an beiden selbst nicht ganz unschuldig ist. Zum einen geht es bei ihm zunächst nicht um eine Beschreibung des tatsächlichen Wissenschaftsprozesses, sondern um eine „Logik der Forschung", d.h. um die Frage nach den theoretischen Grundlagen der Möglichkeit wissenschaftlicher Erkenntnis. Dazu kann die alltägliche Wissenschaftspraxis, wie auch immer sie sich vollzieht, nicht als Gegenargument vorgebracht werden. ${ }^{27)}$ Hier werden die Ebenen methodischer Regeln und die deskriptive Perspektive nicht genügend scharf voneinander getrennt.

[14] Dies gilt für weite Teile der Debatte über den wissenschaftlichen Fortschritt in den sechziger und siebziger Jahren. So will auch TH.S. KUHN (1962) sein wissenschaftshistorisches Werk gelegentlich ins Normative wenden und Ratschläge zur Förderung des wissenschaftlichen Fortschritts geben. Dabei sind die Differenzen in wissenschaftslogischen Fragen zwischen TH.S. KUHN und K.R. POPPER eher gering. ${ }^{28)}$ Andererseits aber hat K.R. POPPER

22. Siehe hierzu die Übersicht bei N. DE MARCH und M. BLAUG (1991, S. 29ff).

23. Siehe hierzu auch D.W. HAND (1985), der ausführt, dass die Anwendung des Ansatzes von I. LAKATOS auf die Entwicklung der Wirtschaftswissenschaften eine Reihe uns wichtiger Einsichten vermittelt hat, darunter insbesondere, dass sie uns gelehrt hat „die richtigen Fragen zu stellen.“ (S. 14).

24. Er verweist dabei auf seine eigene Arbeit R.E. BACKHOUSE (1997, Kapitel 10). - Zur Wissenschaftstheorie der Mathematik siehe I. LAKATOS (1976).

25. Siehe (als Ausnahmen) z.B. S.T. Silva (2009) oder T. GospodAREK (2009).

26. Zu einigen Antwortversuchen darauf siehe B.J. CALDWELL (1991, S. 30f.).

27. Siehe hierzu auch B.J. CALDWELL (1991, S. 4).

28. Siehe hierzu die Bemerkung von TH.S. KuHN (1974, S. 1): „Beinahe immer, wenn wir uns denselben Problemen zuwenden, sind Sir Karls und meine Ansichten über Wissenschaft so gut wie identisch." Und K.R. POPPER (1983, S. XXVII) schreibt: „Und ausserdem stimmen Kuhns und meine Ansichten in der 
(1983, S. XXIff.) durch die Aufzählung von insgesamt 20 ,gelungenen Widerlegungen“ klar gemacht, dass er auch etwas über den tatsächlichen Wissenschaftsprozess aussagen will. Soweit er seine Aussagen auch als empirisch-gehaltvolle Theorien verstanden wissen will, die an der Wirklichkeit scheitern können, muss er sich daher den Einwand gefallen lassen, dass der tatsächliche Wissenschaftsprozess, so wie wir ihn erleben, mit seiner Konzeption - zumindest prima facie - nicht vereinbar ist.

[15] Dieser vermuteten Unvereinbarkeit liegt jedoch ein zweites Missverständnis zugrunde, welches schwerer wiegt: Die Verwechslung der individuellen Motivation der Forscher mit der gesellschaftlichen Organisation der Wissenschaft. Für den Prozess einer rationalen Wissenschaft ist es nicht erforderlich, dass die einzelnen Forscher versuchen, ihre eigenen Hypothesen und Theorien $\mathrm{zu}$ widerlegen. Wichtig ist vielmehr, dass der wissenschaftliche (gesellschaftliche) Diskussionsprozess so ausgestaltet ist, dass solche Widerlegungen möglich sind, und dass Anreize gegeben werden, dass entsprechende Diskussionen stattfinden. Dazu bedarf es eines ,Theorienpluralismus'. Es mag sogar durchaus von Vorteil sein, wenn einzelne Forscher ihre Theorien ,mit Zähnen und Klauen“ verteidigen, weil nur so die Leistungsfähigkeit dieser Theorien voll erkannt werden kann. So hat z.B. I. LAKATOS (1974) darauf hingewiesen, dass es dem wissenschaftlichen Fortschritt durchaus abträglich sein kann, wenn Theorien ,zu schnell' fallen gelassen werden, nachdem empirische Evidenz gegen sie vorgebracht wurde. Wenn man die Konzeption des Kritischen Rationalismus als empirische Theorie auffassen will, dann lässt sich daraus die Hypothese ableiten, dass wissenschaftlicher Fortschritt sich am ehesten dort vollzieht, wo die freie Diskussion Verteidigung und Zurückweisung theoretischer Entwürfe erlaubt. Diese Hypothese dürfte empirisch bewährt sein.

[16] Selbstverständlich versuchen Wissenschaftler auch, ihre eigenen Theorien und Hypothesen zu überprüfen, aber nicht um sie zu widerlegen, sondern um sie ,abzusichern'. Sie tun dies üblicherweise, bevor sie mit einer Hypothese an die (wissenschaftliche) Öffentlichkeit treten. Sie versuchen selbst Gründe zu erwägen, die gegen ihren Ansatz sprechen könnten. Sie werden u.a. Experimente durchführen, statistische Analysen erstellen oder weitere Beobachtungen sammeln. Gerade weil sie ihre Ideen verteidigen wollen, sind sie gezwungen, sie zunächst strengen Tests zu unterwerfen, um dann im öffentlichen Disput nicht widerlegt zu werden. Damit aber hierzu die Motivation vorhanden ist, ist wiederum notwendig, dass der Wissenschaftsbetrieb entsprechend organisiert ist.

[17] Zwischen den Vorstellungen von K.R. POPPER und der alltäglichen Wissenschaftspraxis besteht daher in dieser Hinsicht nicht notwendigerweise ein Widerspruch. K.R. POPPER hat selbst darauf hingewiesen, dass es im Wissenschaftsprozess weniger darum geht, dass die einzelnen Forscher versuchen, ihre Hypothesen und Theorien zu widerlegen, sondern darum, dass dieser Prozess so organisiert wird, dass entsprechende Widerlegungen möglich und sogar wahrscheinlich sind. „Es ist gänzlich verfehlt anzunehmen, dass die Objektivität der Wissenschaft von der Objektivität des Wissenschaftlers abhängt.“ ... „Die Objektivität der Wissenschaft ist nicht eine individuelle Angelegenheit der verschiedenen Wissenschaftler, sondern

Frage der Bedeutung der Falsifikation für die Wissenschaftsgeschichte fast völlig überein." Er listet an dieser Stelle freilich auch die Unterschiede auf, die zwischen seinen Ansichten und denjenigen von TH.S. KUHN bestehen. 
eine soziale Angelegenheit ihrer gegenseitigen Kritik.“ (1962, S. 112.) ${ }^{29)}$ So betrachtet ergibt sich aus der Realität des heutigen Wissenschaftsprozesses nicht notwendigerweise ein Argument gegen die Konzeption von K.R. POPPER und damit auch nicht notwendigerweise dafür, dass die Konzeption wissenschaftlicher Forschungsprogramme ,besser' ist.

\section{Unstrittige Fortschritte im empirisch-methodischen Bereich}

[18] Sieht man einmal von jenen ab. die den empirischen Ansatz generell ablehnen und z.B. in Anlehnung an die Praxeologie des L.V. MISES (1949) auch heute noch glauben, man könne unbestreitbare Aussagen über das Funktionieren des Wirtschaftsprozesses rein logisch ableiten, ${ }^{30)}$ dürfte kaum jemand bestreiten, dass die empirische Ökonomie im Bereich der Methoden in den letzten Jahrzehnten erhebliche Fortschritte gemacht hat. Sie haben dazu geführt, dass es heute in den Sozialwissenschaften neben dem ,theoretischen Imperialismus' des ökonomischen Verhaltensmodells auch insofern einen Imperialismus der Ökonomen gibt, als sie mit ihren hoch entwickelten empirischen Methoden Fragestellungen aus ganz anderen Sozialwissenschaften bearbeiten, teilweise ohne besondere Kenntnisse der theoretischen Grundlagen dieser Wissenschaften.

[19] Als mit dem Aufkommen (relativ) leistungsfähiger Rechner in den siebziger Jahren des vergangenen Jahrhunderts und der Entwicklung der entsprechenden Software umfangreichere empirische Untersuchungen überhaupt erst möglich wurden, gab es beim empirischen Arbeiten eine relativ klare Arbeitsteilung zwischen Ökonomen einerseits und anderen Sozialwissenschaftlern, insbesondere Soziologen und Psychologen andererseits: Ökonomen verwendeten Zeitreihendaten, die im Wesentlichen aus der amtlichen Statistik kamen, während die anderen Sozialwissenschaftler vor allem Querschnittsdaten verwendeten, welche sie mit Umfragen oder Experimenten erhoben hatten. ${ }^{31)}$ Damit hatten die Ökonomen praktisch ausschliesslich Aggregatdaten zur Verfügung, während die übrigen Sozialwissenschaftler sich vorwiegend mit Individualdaten befassten. In diese Richtungen wurden auch die Methoden weiterentwickelt, was sich in den entsprechenden Lehrbüchern niederschlug. ${ }^{32)}$

[20] Dies änderte sich, als Wirtschaftsforschungsinstitute begannen, Unternehmensdaten (und somit ebenfalls Individualdaten) zu erheben. In Deutschland spielte hier insbesondere das Ifo Institut für Wirtschaftsforschung in München mit seinem Konjunkturtest eine herausragende Rolle, während in der Schweiz vor allem die Konjunkturforschungsstelle an der ETH Zürich (KOF), die analoge Daten für die Schweiz erhob, bedeutsam war. Damit aber wurde es erforderlich, dass sich auch Ökonometriker mit den Problemen der Analyse von Individual-

29. Siehe hierzu auch B.J: CALDWELL (1991, S. 25ff.).

30. Siehe TH. PolleIT (2009), der argumentiert: „Aufbauend auf Anerkennung von absolut wahrem Wissen ist wissenschaftlicher Fortschritt möglich.“ Er präsentiert fünf ökonomische Aussagen, für die er - ohne Einschränkung - Wahrheit reklamiert.

31. Gelegentlich wurden beim gleichen Personenkreis auch mehrere Umfragen hintereinander durchgeführt, womit die ersten Panels entstanden. Diese blieben jedoch auf zunächst wenige Zeitpunkte beschränkt.

32. Siehe z.B. H. THEIL (1971) als prominentes Ökonometriebuch der damaligen Zeit oder H.M. BLALOCK (1971), in welchem sich wesentliche Verfahren finden, die damals in den anderen Sozialwissenschaften angewendet wurden. 
daten beschäftigten, was zur Entwicklung der Mikroökonometrie führte. ${ }^{33)}$ Dabei rückten neben reinen Querschnittsanalysen immer mehr auch Panelanalysen ins Zentrum der Aufmerksamkeit, wobei die zeitliche Dimension gegenüber früher deutlich an Bedeutung gewann. Dies war u.a. auch dann unvermeidbar, wenn (z.B. im Bereich der Arbeitsmarktforschung) individuelle ,Karrieren` zu verfolgen waren.

[21] Seit den achtziger Jahren erlebte die Mikroökonometrie einen ungeheuren Aufschwung, dem die Statistiker aus den anderen Sozialwissenschaften kaum folgten. Ihre verbreitete Anwendung wurde auch dadurch gefördert, dass (in Zusammenarbeit mit anderen Sozialwissenschaftlern) grosse Datensätze zur Erfassung der wirtschaftlichen und gesellschaftlichen Situation der Bevölkerung erhoben wurden, welche die Untersuchung ganz neuer Fragestellungen ermöglichten. In Deutschland ist dies das sozioökonomische Panel (SOEP), in dessen Rahmen seit 1984 jährlich eine repräsentative Stichprobe der deutschen Bevölkerung befragt wird, die heute jedes Jahr mehr als 11'000 Haushalte mit mehr als 20'000 Personen umfasst. In der Schweiz startete die entsprechende Unternehmung, das Swiss Household Panel (SHP), mit gebührender Verspätung erst 1999, im Jahr 2005 umfasste es 6'800 Haushalte mit beinahe $17^{\prime} 000$ Personen. ${ }^{34)}$ Auf internationaler Ebene gibt es heute u.a. das International Social Survey Programme (ISSP), das World Value Survey (WVS), ${ }^{35)}$ (mit speziellem Focus auf die schulische Ausbildung) das OECD Programm für International Student Assessment (PISA) ${ }^{36)}$ sowie (für Europa mit einem speziellen Focus auf Probleme des Alters und der Gesundheit) SHARE. ${ }^{37)}$ Mit Ausnahme von SHARE waren die Ökonomen bei der Erhebung dieser Daten meist nicht federführend, wohl aber häufig bei den mit diesen Daten durchgeführten empirischen Untersuchungen sowie insbesondere bei der Entwicklung der hierzu notwendigen statistisch-ökonometrischen Verfahren. Dies wurde u.a. auch dadurch dokumentiert, dass JAMES J. HeCKMANN und DANIEL LEIGH MCFADDEN im Jahr 2000 für ihre entsprechenden Arbeiten den Nobelpreis für Ökonomie erhielten.

[22] Diese Verfahren können prinzipiell auf alle Fragestellungen angewendet werden, die mit Hilfe von Mikrodaten untersucht werden können, unabhängig vom Bereich, welchem das zu untersuchende Problem inhaltlich zugeordnet ist. Dass sie im Bereich der Arbeitsmarktökonomik Anwendung finden, mag wenig erstaunen, schliesslich ist dies ein traditioneller Bereich der Wirtschaftswissenschaften, und ausserdem gibt es hier ein umfangreiches, durch die Arbeitsämter offiziell erhobenes Datenmaterial. Insofern kann man hier auch nicht von Imperialismus sprechen. Anders sieht es dagegen z.B. bei der ,Economics of Education“ aus, einem neu aufstrebenden Gebiet, in welchem Ökonomen heute Fragestellungen untersuchen,

33. Das erste Lehrbuch hierzu im deutschsprachigen Raum dürfte G. RoNNING (1991) sein.

34. Zum SOEP siehe http://www.diw.de/de/diw_02.c.221178.de/ueber_uns.html, zum SHP http://www. swisspanel.ch/?lang=de $(12 / 02 / 11)$.

35. Zum ISSP siehe http://www.issp.org/ (09/03/11), zum WVS http://www.worldvaluessurvey.org/ (09/03/11) sowie R. INGLEHART et al. (2004).

36. Siehe hierzu http://www.pisa.oecd.org/document/58/0,2340,en_32252351_32236159_33688954_1_1_1_ 1,00.html (09(03/11).

37. Survey on Health, Ageing and Retirement in Europe; siehe hierzu A. BörSCH SuPAN und H. JÜRGES (2005), A. BÖRSCH SUPAN et al. (2005) sowie http://www.share-project.org/ (11/11/10). 
die früher praktisch ausschliesslich von Erziehungswissenschaftlern behandelt wurden. So untersucht A. LEON (2004) z.B. den Einfluss der Erziehung auf die Fertilität, M. ZAvODNY (2006) fragt, in wieweit der Fernsehkonsum die schulischen Leistungen von Kindern beeinflusst. F. HofFMANN und P. ORePOUlos (2009) befassen sich damit, welchen Einfluss die Qualität der Professoren auf die Examensergebnisse ihrer Studierenden hat. Und während M.R. WeST und L. WOESSMANN (2010) anhand der PISA-Daten untersuchen, welchen Einfluss der Anteil privater Schulen in einem Land auf die Ergebnisse in Lesen, Mathematik und Naturwissenschaften hat, befassen sich B. MuelLER und ST.C. Wolter (2011) mit den Auswirkungen der statistischen Diskriminierung beim Übergang von der Schule ins Berufsleben Selbstverständlich sind Antworten auf solche Fragen auch für Ökonomen interessant. Dennoch bleibt festzustellen, dass sich noch vor nicht all zu langer Zeit Ökonomen kaum an die wissenschaftliche Untersuchung solcher Fragen herangewagt hätten. Hier hat sich ein dramatischer Wandel vollzogen.

[23] Dies ist nicht auf eine irgendwie geartete (oder auch nur behauptete) theoretische Überlegenheit des ökonomischen Ansatzes zurückzuführen; schliesslich spielen theoretische Überlegungen in vielen dieser Arbeiten bestenfalls eine untergeordnete Rolle. Das Erstaunliche ist vielmehr, dass die anderen Sozialwissenschaften, die hier einen erheblichen theoretischen Vorsprung haben sollten, diesen anscheinend nicht ausnützen können. Der methodische Vorsprung der Ökonomen dominiert hier offensichtlich den theoretischen Vorsprung der anderen Sozialwissenschaftler.

[24] Die derzeitige Situation mag freilich nur von kurzer Dauer sein. Zwar ist nicht davon auszugehen, dass sich die Ökonomen aus diesem Untersuchungsfeld wieder gänzlich zurückziehen werden; sie werden im Gegenteil in Zukunft möglicherweise noch stärker in dieses und andere ähnliche Felder hineingehen. Hierfür sorgen schon der Zwang zur Publikation, dem junge Wissenschaftler heute sehr viel stärker als früher ausgesetzt sind, und die sich daraus ergebende Suche nach immer neuen Fragestellungen. Andererseits sollte es möglich sein, innerhalb weniger Jahre auch in den anderen Sozialwissenschaften genügend Forscher so weit in den modernen mikroökonometrischen Verfahren auszubilden, dass der methodische Vorsprung der Ökonomen schwindet oder vielleicht ganz verloren geht. Schliesslich ist an diesen Verfahren nichts spezifisch, ökonomisch ${ }^{\text {(38) }}$ Und wenn die anderen Sozialwissenschaftler mit den Ökonomen methodisch gleich gezogen haben, sollten sie ihren theoretischen Vorsprung sowie ihre besseren institutionellen Kenntnisse ausnutzen können. Dies setzt freilich voraus, dass der Ausbildung mit statistischen Verfahren in diesen Wissenschaften in Zukunft ein deutlich höheres Gewicht beigemessen wird als heute. Während dies im angelsächsischen Raum mit ziemlicher Sicherheit geschehen wird, bleibt abzuwarten, ob es auch im deutschsprachigen Raum der Fall sein wird. Die Aversion gegen die ,Quantifizierung', die viele Vertreter dieser Wissenschaften hegen, dürfte dieser Entwicklung eher hinderlich sein. ${ }^{39)}$ Damit ist in diesem Bereich noch für längere Zeit die Möglichkeit einer Hegemonie der Ökonomen gegeben.

38. Soweit diese Arbeiten auf einer expliziten theoretischen Grundlage aufbauen, sind sie freilich in dem Sinn ,ökonomisch‘, als sie das ökonomische Verhaltensmodell zugrunde legen.

39. Siehe z.B. das Manifest der SAGW (2010). 


\section{Wissenschaftliche Revolutionen in den Wirtschaftswissenschaften}

[25] Folgt man der Terminologie von TH.S. KuHN (1962), dann sollte man diese Entwicklung wohl in die Kategorie ,Normalwissenschaft" einordnen, auch wenn dabei ausgesprochen interessante und auch politisch relevante Ergebnisse erzielt werden. Die Frage ist jedoch, ob es daneben auch Entwicklungen gab, die man als ,wissenschaftliche Revolutionen ' bezeichnen könnte. Als Ökonomen begannen, diese Frage zu diskutieren, stand (entsprechend der damaligen Zeit) die keynesianische Revolution im Vordergrund. ${ }^{40)}$ Die Entwicklung der davor nicht existierenden - makroökonomischen Theorie konnte man im Vergleich zum davor existierenden Theoriegebäude durchaus als Revolution begreifen. Schliesslich hat sie zur ,Neoklassischen Synthese' geführt, welche die Lehrbücher nach dem Zweiten Weltkrieg dominiert hat. Dementsprechend sprach man auch von der monetaristischen Gegenrevolution in Zusammenhang damit, dass sich in Geldtheorie und Geldpolitik in den siebziger Jahren weitgehend die Iden der Monetaristen durchsetzten, die später in Verbindung mit der Theorie rationaler Erwartungen zur ,Neuen klassischen Makroökonomik' führte. Worin aber bestanden diese Revolutionen eigentlich, und inwieweit kann man aus heutiger Perspektive hier von Revolutionen sprechen ${ }^{41)}$

[26] Interessant ist in diesem Zusammenhang die Position von M. BRONFENBRENNER (1971, S. 138). ${ }^{42)}$ Er sieht drei Revolutionen in der Entwicklung der ökonomischen Theorie: eine Laisser-faire Revolution, die üblicherweise mit A. SMITH (1776) in Beziehung gesetzt wird, seiner Meinung nach aber auf D. HuME (1752) zurückgeht, die marginalistische Revolution in der zweiten Hälfte des 19. Jahrhunderts, die er auf das Jahr 1870 datiert, und die keynesianische Revolution, ausgelöst durch J.M. KEYNES (1936). ${ }^{43)}$ Interessant ist, wie sich in diesen ,Revolutionen' politische Ideologien, welche die Rolle des Staates betreffen, und methodische Fortschritte verbinden. Die Laissez-Faire Revolution war in erster Linie eine Veränderung des politischen Standpunkts; der Staat sollte aus dem Wirtschaftsleben weitgehend zurückgedrängt werden. Die marginalistische Revolution war zweifellos in erster Linie eine methodische Angelegenheit. Dennoch sind auch hier politische Positionen im Spiel, gegen welche die neue Theorie aufgebaut wurde. Dies gilt nicht nur für den Marxismus, sondern z.B. auch für die Deutsche Historische Schule. ${ }^{44)}$ Auch bei Keynes finden wir wieder beide Elemente: Während die wissenschaftliche Innovation vor allem darin bestand, dass man Aggregate und nicht mehr einzelne Individuen betrachtete (und damit die moderne makroökonomische Theo-

40. Siehe z.B. M. BlaUG (1976, S. 34; 1990, S. 88) sowie die dort angegebenen Hinweise.

41. Auch wenn sie nie in diesem Zusammenhang diskutiert wurde, könnte man die institutionelle Wende in der Ökonomik, die eine zumindest partielle Abkehr von dem bereits von H. ALBERT (1963) kritisierten ,Modellplatonismus' der neoklassischen Theorie brachte, mindestens mit gleichem Recht als wissenschaftliche Revolution betrachten. Zu dieser,Wende‘ siehe H. ALBERT (2006, S. 113ff.).

42. Die gegenwärtige Situation, d.h. die Situation zu Beginn der siebziger Jahre, sieht er als ,,vorrevolutionär“ an. - Zu ,Revolutionen“ in den Wirtschaftswissenschaften siehe auch J. HicKS (1976).

43. Er merkt freilich auch an, dass sich keine dieser Revolutionen mit den grossen Revolutionen in den Naturwissenschaften, der Kopernikanischen, der Newtonschen oder der Darwinschen Revolution vergleichen kann. (S. 139.)

44. Zur Deutsche Historischen Schule siehe z.B. P.R. SENN (2006). 
rie überhaupt erst begründete), war der zentrale (wirtschafts-)politische Streitpunkt, inwiefern der Staat in den Wirtschaftsablauf sinnvoll eingreifen kann bzw., falls er das kann, ob er das auch soll. Auch die jüngeren ,Revolutionen', die monetaristische Gegenrevolution oder die Revolution der rationalen Erwartungen, drehen sich letztlich immer auch (und gelegentlich sogar vorwiegend) um diese Frage. Dies gilt selbst dann, wenn methodische Fragen im Vordergrund der Diskussion stehen. Ein Indikator dafür, welche wirtschaftspolitische Position eingenommen wird, ist, inwieweit die Modelle neben klassischer, d.h. freiwilliger, auch unfreiwillige Arbeitslosigkeit kennen.

[27] Dass man nicht nur Einzelpersonen betrachtet, sondern Aggregate in das Zentrum der Analyse stellt, war sicher neu und ist ein kaum bestrittenes Verdienst des keynesianischen Ansatzes. Dabei wird zwar mit mikroökonomischen Plausibilitätsargumenten gearbeitet, aber es gibt (noch) keine mikroökonomische Fundierung, d.h. die Aussagen der Theorie basierten zumindest nicht explizit auf der Annahme individuell rationalen Verhaltens der Wirtschaftssubjekte. Dies wird auch von M. BLAUG (1976) betont. ${ }^{45)}$ Tatsächlich gab es noch in den siebziger Jahren eine Diskussion darüber, inwieweit die makroökonomische Theorie überhaupt einer Mikrofundierung bedarf. ${ }^{46)}$ Rein logisch gesehen bedarf es einer solchen ,Mikrobasis', d.h. einer durch mikroökonomische Entscheidungskalküle gelegten Grundlage der makroökonomischen Relationen, nicht, um eine aussagekräftige makroökonomische Theorie zu entwickeln. Empirisch gehaltvolle Theorien, die dem deduktiv-nomologischen Erklärungsmodell folgen, lassen sich auch ohne sie konstruieren. So wurden noch vor wenigen Jahrzehnten auf Plausibilitätsargumenten beruhende Makrorelationen akzeptiert, solange sie mit Hilfe ökonometrischer Methoden statistisch abgesichert schienen. Dies dürfte sich im Bereich der Theorie inzwischen weitgehend erledigt haben: Unabhängig von ideologischen Gegensätzen verwenden heute sowohl die (eher links orientierte) ,Neue (keynesianische) Makroökonomik' als auch die (eher konservativ orientierte) ,Neue klassische Makroökonomik ${ }^{47}$ (bzw. die entsprechenden ,Neo-Neo-...-Ansätze‘) heute eine solche ,Mikrobasis‘.

[28] Im Vertrauen auf die Existenz und Stabilität solcher Beziehungen wurden auch wirtschaftspolitische Maßnahmen getroffen. Das berühmteste Beispiel für eine solche Beziehung dürfte die modifizierte Phillips-Beziehung sein, jene (behauptete) langfristige Beziehung zwischen Inflation und Arbeitslosigkeit, die zudem (zumindest) implizit in fast allen großen makroökonometrischen Modellen der siebziger Jahre enthalten war. ${ }^{48)}$ Sie wurde zuerst von A.W. PHILLIPS (1958) als Beziehung zwischen Arbeitslosigkeit und Nominallohnentwicklung, entdeckt' und später zu einer Beziehung zwischen Inflation und Arbeitslosigkeit erweitert. Im Jahr 1960 wurde sie von P.A. SAMUELSON und R.M. SoLOW als „Speisekarte der Wirtschaftspolitik“ bezeichnet. Solche - nach ihrem ,Entdecker' benannten - ,Phillips-Kurven“ wurden für eine ganze Reihe von Ländern ökonometrisch geschätzt, und gestützt auf diese empirische

45. Zur rudimentären Mikrofundierung der keynesianischen Theorie siehe auch z.B. A. LEJONHUFVUD (1976, S. 105).

46. Siehe hierzu z.B. H.J. RAMSER (1987, S. 8ff.) sowie F. MACHLuP (1960) und E. SCHLICHT (1977).

47. Zur Einführung in die neue keynesianische (Makro-)Ökonomik siehe R.J. GORDON (1990) oder D. ROMER (1993). Zur Gegenüberstellung beider Ansätze siehe H.J. RAMSER (1987) sowie N.J. MANKIW (1990).

48. Siehe hierzu G.C. Chow und S.B. Megdal (1978). 
Evidenz glaubte man, durch eine einmalige Erhöhung der Inflationsrate die Arbeitslosenquote dauerhaft senken zu können. Die in den siebziger und achtziger Jahren darauf aufbauende Politik muss jedoch als gescheitert betrachtet werden: Die Erhöhung der Inflationsrate mag zwar kurzfristig zu einer Erhöhung der Beschäftigung beigetragen haben, aber bereits mittelfristig liess sich die Arbeitslosigkeit dadurch nicht senken, sondern es trat Stagflation ein, wie es der von M. FRIEDMAN (1968) vorgestellten Hypothese der natürlichen Rate der Arbeitslosigkeit entspricht. Wie J. ROBINSON bereits 1972 schrieb: „Die experimentelle Demonstration der Phillipskurve hat ... versagt.“ (S. 47) Nicht zuletzt dieses praktische Scheitern der Makroökonomik hat - zusammen mit der schlechten Prognoseleistung geschätzter makroökonometrischer Grossmodelle ${ }^{49)}$ dazu geführt, dass die Notwendigkeit einer Mikrofundierung heute kaum mehr bestritten wird.

[29] Betrachtet man vor diesem Hintergrund die methodischen Aspekte der keynesianischen Revolution, kann man feststellen, dass sich das Argumentieren in Aggregaten durchgesetzt hat, der Verzicht auf eine Mikrobasis dagegen nicht. Insofern war diese Revolution nur zur Hälfte erfolgreich. Dies ist anders bei der marginalistischen Revolution. Der Übergang von der Durchschnittsbetrachtung, wie sie noch von KARL MARX insbesondere auch im Kapital angestellt wurde, zur Durchschnittsbetrachtung, die mit den Namen LEON WALRAS, ALFRED Marshall und CARL Menger verbunden ist, hat die ökonomischen Argumentationen grundlegend verändert und wird auch nirgends bestritten. Betrachtet man den methodischen Aspekt in der Theoriebildung, dann war dies die wohl mit Abstand bedeutendste (und möglicherweise sogar die einzig wirkliche) wissenschaftliche Revolution in den modernen Wirtschaftswissenschaften. Daneben kann nur noch die keynesianische Revolution einen - wenn auch vermutlich weniger bedeutsamen - grundlegenden Beitrag zur Methodik der ökonomischen Theoriebildung für sich beanspruchen.

[30] Bei allen übrigen ,Revolutionen' ging es vor allem um empirische Fragen, die freilich zumeist wirtschaftspolitisch und/oder ideologisch erhebliche Implikationen hatten. Dies gilt z.B. für die monetaristische Gegenrevolution, in der es zunächst darum ging, ob die Fiskaloder die Geldpolitik ein wirksameres Mittel staatlicher Wirtschaftspolitik sein kann, inwieweit sie überhaupt sinnvoll eingesetzt werden können und wie man der damaligen Inflation wirksam begegnen kann. Die Antwort von M. FRIEDMAN $(1948,1968)$ war eindeutig: ${ }^{50)}$ (i) Inflation ist im Wesentlichen ein monetäres Phänomen; sie kann und soll über die Geldmenge gesteuert werden. (ii) Die Geldpolitik ist zwar wirksamer, aber kaum steuerbar, und deshalb sollte sich die staatliche Wirtschaftspolitik darauf beschränken, die Geldmenge mit einer festen Rate wachsen zu lassen und in der Finanzpolitik ein ausgeglichenes Budget (mit möglichst geringen Steuern) vorzulegen. Dies war zwar inhaltlich ein völlig konträres Rezept zum keynesianischen Ansatz, aber die methodischen Unterschiede waren, soweit sie überhaupt vorhanden waren, eher gering.

49. So haben C.W.J. GRANGER und P. NEWBOLD (1975) gezeigt, dass mit (,untheoretischen') einfachen Modellen der univariaten Zeitreihenanalyse erstellte Prognosen über die zukünftige wirtschaftliche Entwicklung häufig besser abschnitten als Prognosen, die mit Hilfe großer makroökonometrischer Modelle erstellt wurden und die für sich in Anspruch nahmen, auf der ökonomischen Theorie zu basieren.

50. Siehe hierzu auch M. FrIEDMAN und A. SCHWARZ (1965). 
[31] Sie bezogen sich bestenfalls auf die Frage, inwieweit sich die Individuen rational verhalten. Dies wurde aufbauend auf der Theorie Rationaler Erwartungen im Sinne von J. MuTH (1961) in der zweiten Welle der monetaristischen Revolution thematisiert, die zur Neuen Klassischen Makroökonomik bzw. zur „Ökonomie des Dr. Pangloss“ führte, wie sie (aus eher keynesianischer Perspektive) von W.H. BUITER (1980) bezeichnet wurde. Danach sind die Möglichkeiten staatlicher Eingriffe in den Wirtschaftsablauf noch begrenzter, als M. FRIEDMAN (1968) zuvor angenommen hatte. Tatsächlich wurde hier ein Problem der keynesianischen Ansätze aufgedeckt: Sie waren (bis zu diesem Zeitpunkt) damit vereinbar, dass sich die Bürgerinnen und Bürger durch die staatliche Wirtschaftspolitik längerfristig systematisch täuschen lassen. Die Tatsache, dass die Annahme rationaler Erwartungen bald auch in die Modelle der neuen keynesianischen Makroökonomik eingebaut werden konnte, ohne dass dies zu den gleichen wirtschaftspolitischen Abstinenzempfehlungen führte, zeigt freilich, dass es sich hier zwar um ein zentrales wirtschaftspolitisches, aber nicht um ein grösseres methodisches Problem handelt.

[32] Insgesamt betrachtet ergibt sich daraus, dass das Konzept der wissenschaftlichen Revolution für die Erklärung der Entwicklung der wirtschaftswissenschaftlichen Theorien nur wenig hergibt. Fragt man nach den grösseren methodischen Umwälzungen, kann man bestenfalls zwei Beispiele finden. Dazu kommt, dass die Inkommensurabilitätsthese in diesen beiden Beispielen nicht gilt: So wurde z.B. für die auf dem klassischen Ansatz basierende Theorie von K. MARX gezeigt, wie sie im neoklassischen Modell als ein Spezialfall dargestellt werden kann, der unter bestimmten Annahmen gilt. ${ }^{51)}$ Man dürfte daher nicht ganz falsch liegen, wenn man vermutet, dass der Begriff der Revolution in den Wirtschaftswissenschaften eher anderen Intentionen dient, seien diese z.B. rhetorischer, ideologischer oder allgemeinpolitischer Natur, als dem Aufzeigen massgeblicher Fortschritte in der ökonomischen Theoriebildung. Dies gilt auch für den Wechsel im Paradigma, der damit verbunden sein soll: Er bezog sich jeweils weniger auf die theoretische Perspektive als vielmehr auf die Einschätzung der wirtschaftspolitischen Handlungsmöglichkeiten, auch wenn selbstverständlich wissenschaftliche Argumente als Begründung herangezogen wurden. ${ }^{52)}$

\section{Das Paradigma der Wirtschaftswissenschaften}

[33] Damit stellt sich die Frage, inwieweit es überhaupt Paradigmen in den Wirtschaftswissenschaften gibt und wo diese allenfalls angesiedelt sein könnten. Entsprechend den obigen Ausführungen über die Entwicklung der makroökonomischen Theorie dürfte es sehr problematisch sein, dort von Paradigmen zu sprechen, zumindest solange man den hohen Anspruch damit verknüpfen will, den TH.S. KUHN (1962) damit verbindet und an seinen Beispielen aus den Naturwissenschaften aufzeigt. Wenn man in den Wirtschaftswissenschaften überhaupt sinnvoll von einem Paradigma reden will, dürfte dies im Bereich der mikroökono-

51. Siehe hierzu z.B. N. OKISHIO (1963) sowie die Verweise in J. KORNAI (1971, S. 359).

52. Entgegen den Vermutungen von TH.S. KUHN (1962) und P.K. FEYERABEND (1976) dürfte die Inkommensurabilitätsthese freilich auch in den Naturwissenschaften nicht haltbar sein. Siehe hierzu G. ANDERSSON (1988, S. 110ff.). 
mischen Theorie angesiedelt sein. Dort findet sich tatsächlich eine weitestgehend geteilte methodische Basis, die man als solche betrachten kann.

[34] Es handelt sich um den Kern des ökonomischen Verhaltensmodells: den methodologischen Individualismus und die sich daraus ergebenden Konsequenzen für die Erklärung makroökomischer Phänomene. Gesellschaftliche Phänomene werden als (in aller Regel nicht intendierte) Ergebnisse individueller Handlungen begriffen: Die Individuen entscheiden sich bei aller Begrenztheit der ihnen zur Verfügung stehenden Informationen in ihrem Handeln ,rational' zwischen denen ihnen bekannten Handlungsalternativen; sie schätzen deren Vorund Nachteile und entscheiden sich gemäss ihrem relativen Vorteil. Dabei wird - zumindest im Prinzip - zwischen den Intentionen bzw. Präferenzen der Individuen und ihren Handlungsmöglichkeiten strikt unterschieden. ${ }^{53)}$

[35] Rationalität bedeutet hier nicht, dass das Individuum in jedem Augenblick optimal handelt, sondern lediglich, dass es prinzipiell in der Lage ist, gemäß seinem relativen Vorteil zu handeln, d.h. seinen Handlungsraum abzuschätzen und zu bewerten, um dann entsprechend zu handeln. Dabei ist zu berücksichtigen, dass das Individuum sich immer unter unvollständiger Information entscheiden muss und dass die Beschaffung zusätzlicher Information Kosten verursacht. Auch muss es häufig unter Zeitdruck entscheiden. Kosten zur Informationsbeschaffung wird das Individuum vor allem dann auf sich nehmen, wenn es eine relevante Veränderung seines Handlungsraumes (und/oder seiner Präferenzordnung) registriert und daher eine neue Abschätzung und Bewertung seiner Handlungsmöglichkeiten vornehmen muss. Auf eine solche Veränderung reagiert ein rationales Individuum ,systematisch', d.h. nicht zufällig oder willkürlich, aber auch nicht dadurch, dass es sich unabhängig von diesen Veränderungen streng an vorgegebene Regeln hält. Damit kann dieses Verhalten durch Setzung von Anreizen systematisch beeinflusst werden, wobei sich solche Anreize im Wesentlichen aus Veränderungen des Handlungsraums der Individuen (der Restriktionen) ergeben. Damit sind auch Prognosen von Verhaltensänderungen als Reaktion auf Veränderungen des Handlungsspielraumes möglich.

[36] Das hier unterstellte ,schwache Rationalitätsprinzip' hat für die Sozialwissenschaften einen bedeutenden Stellenwert. ${ }^{54)}$,Verstehen' menschlichen Handelns ist erst möglich, wenn man die im ökonomischen Verhaltensmodell angelegte Unterscheidung zwischen Präferenzen und Restriktionen (Zielen und Mitteln) akzeptiert und außerdem davon ausgeht, dass die Individuen die ihnen zur Verfügung stehenden Mittel (rational) zur Erreichung ihrer Ziele einsetzen, wobei natürlich die subjektiven Perzeptionen eine wichtige Rolle spielen. Diese Rationalitätsannahme ist in diesem Sinne keine empirische Annahme, sondern eine Voraussetzung dafür, dass ich menschliches Handeln in einer bestimmten Weise analysieren kann. ${ }^{55)}$ Begreift

53. Zur ausführlichen Darstellung dieses Ansatzes siehe G. KIRCHGÄSSNER (1991).

54. Siehe hierzu G. KIRCHGÄSSNER (2004).

55. Man kann die Rationalitätsannahme und - darauf aufbauend - das ökonomische Verhaltensmodell freilich auch als eine prinzipiell empirisch überprüfbare Hypothese auffassen; wegen der großen Allgemeinheit dieser Hypothese dürfte die Überprüfung im Allgemeinen aber recht schwer fallen. Überprüfungen sind jedoch grundsätzlich (und oft auch recht leicht) bei genauerer Spezifikation der Restriktionen oder der Präferenzen, d.h. bei Hinzufügen zusätzlicher Hypothesen möglich. Dann aber werden ,verbundene Hypothesen“ getestet, so dass nicht ohne weiteres ausgemacht werden kann, ob im Falle einer Ablehnung die Rationali- 
man dieses Verhaltensmodell in der hier vorgeschlagenen Weise als Voraussetzung zum Verstehen menschlichen Handelns, so verschwindet auch der Unterschied zwischen ,Verstehen“ und ,Erklären': Ich kann menschliches Handeln nur verstehen, wenn ich es mit Hilfe eines solchen Modells rationalen Verhaltens erklären kann. ${ }^{56)}$

[37] Dabei interessiert jedoch weniger das Verhalten einzelner Individuen als vielmehr das Verhalten größerer Gruppen von Individuen, der ,Aggregate': Nicht das Verhalten eines bestimmten einzelnen Individuums ist interessant, sondern das ,typische“ Verhalten, d.h. Regelmäßigkeiten im Verhalten aller oder zumindest einer Mehrheit der betrachteten Individuen in der jeweils untersuchten Gruppe. Die Mikrotheorie bietet hier (nur) die Basis, um Makrophänomene erklären zu können. Werden nämlich durch eine Veränderung einer bestimmten Makrovariablen die Rahmenbedingungen für das Handeln aller Individuen bzw. einer bestimmten Gruppe in ähnlicher Weise beeinflusst, so kann man davon ausgehen, dass deren Reaktion zwar nicht unbedingt in jedem Einzelfall, aber im Durchschnitt jene Regelmäßigkeit aufweist, welche aus dem individuellen Entscheidungskalkül heraus erklärt werden kann.

[38] Die Betrachtung von Aggregaten ist noch aus einem anderen Grund zentral für die Sozialwissenschaften: Nur so können von den einzelnen Individuen nicht intendierte soziale Folgen individueller Handlungen erfasst werden, welche zu einer spontanen Ordnung führen. Dies aber ist eine zentrale Aufgabe der Sozialwissenschaften, nach Ansicht vieler Autoren sogar die zentrale Aufgabe. Für die Ökonomie ist dies fast trivial und seit ADAM SMITH (1776) eigentlich selbstverständlich: Üblicherweise hat keines der Individuen, die auf einem Markt tätig sind, die Absicht, dadurch den Marktmechanismus als einen gesellschaftlichen Koordinationsmechanismus in Gang zu setzen, und doch tragen alle, bewusst oder unbewusst, mit oder gegen ihre Absicht, dazu bei. Aber nicht nur das Funktionieren des Marktmechanismus ergibt sich als nicht intendierte Nebenfolge von Handlungen vieler Individuen, gleiches gilt auch für politische Mechanismen. Und auch viele andere Institutionen ergeben sich daraus.

[39] Dass dies das zentrale Paradigma der Wirtschaftswissenschaften ist, dürfte heute innerhalb der Wirtschaftswissenschaften kaum bestritten werden. Mit dem Aufkommen der makroökonomischen Theorie war es, wie oben ausgeführt wurde, jedoch zeitweise bestritten. Spätestens seit auch die neue keynesianische Makroökonomik mit einer Mikrofundierung arbeitet, hat sich dies jedoch erledigt. Dies heisst freilich nicht, dass dieser Ansatz in den Sozialwissenschaften unbestritten wäre; es gibt als Alternative z.B. die Möglichkeit einer Sozialwissenschaft in der Tradition von E. DuRKHEIM (1895, S. 193), der forderte, Soziales nur durch Soziales zu erklären. Insbesondere im Bereich der Soziologie gibt es nach wie vor theoretische Ansätze, die ausschliesslich auf der Aggregatsebene argumentieren und für sich behaupten, einer Mikrobasis nicht zu bedürfen. Zwar hat H. ESSER (1993) am Beispiel der Phänomenologie gezeigt, dass eine Rekonstruktion dieses Ansatzes im Rahmen des ökonomi-

tätsannahme und damit das zugrunde liegende Verhaltensmodell oder ob die zusätzlichen speziellen Annahmen verworfen werden müssen.

56. Genau betrachtet handelt es sich hier um das allgemeine Grundmodell einer verstehenden Sozialwissenschaft im Sinn von MAX WEBER. Siehe hierzu z.B. die Beschreibung dieses Ansatzes in M. WEBER (1913, S. 429). 
schen Verhaltensmodells möglich ist, aber dies muss nicht allgemein gelten, und es ist diskutabel, in welchen Bereichen dieser Ansatz fruchtbar ist.

[40] Andererseits kann man festhalten, dass der ökonomische Ansatz heute auch in den anderen Sozialwissenschaften verwendet wird, auch wenn er dort wesentlich umstrittener als in den Wirtschaftswissenschaften ist. Dies gilt insbesondere für die Politikwissenschaft, aber auch für die Soziologie. Der ,ökonomische Imperialismus' hat dort viele Unterstützer, aber auch nicht wenige Gegner gefunden. ${ }^{57)}$ Im Sinne eines den Fortschritt in den Wissenschaften fördernden Theorienpluralismus kann dies sinnvoll sein; das Problem im Wettbewerb der Ideen ist hier jedoch, dass die Gruppe jener, die eine Anwendung des ökonomischen Ansatzes in ihrem Bereich nicht als sinnvoll erachten, stark fragmentiert ist, so dass es sehr viel schwieriger als im Bereich der Wirtschaftswissenschaften ist, zu eruieren, welches ihr Paradigma ist. Von einem gemeinsamen Paradigma kann in diesem Bereich kaum die Rede sein.

[41] Damit bleibe die Frage der von Th.S. KuHn (1962) und P.K. FeYERABEND (1976) immer wieder behaupteten Inkommensurabilität zwischen den verschiedenen wissenschaftlichen Ansätzen. Ist sie in den Wirtschaftswissenschaften (oder allgemeiner in den Sozialwissenschaften) zu beobachten? Es ist nicht zu übersehen, dass zum einen Wissenschaftler verschiedener Disziplinen (in den Sozialwissenschaften) gelegentlich (oder vielleicht auch häufig) aneinander vorbei reden, und dass sie zweitens auch gegenüber dem gleichen Gegenstand gelegentlich sehr unterschiedliche Perspektiven einnehmen. Beides aber hat nichts mit Inkommensurabilität zu tun. Selbst dort, wo es Inkommensurabilität ex ante geben sollte, dürfte sie kaum jemals ex post gegeben sein: Wenn zwei Theorien aufgrund ihrer unterschiedlichen Perspektiven zu unterschiedlichen, möglicherweise auch gegenteiligen Aussagen kommen, besteht grundsätzlich die Möglichkeit nachzuvollziehen, welches die entscheidenden Annahmen sind, die dies bewirken. Man wird sich vielleicht nicht darüber einigen können, welches die sinnvolleren Annahmen bzw. welches das besser geeignete Modell ist, um ein bestimmtes Phänomen zu erklären, aber gerade die Auseinandersetzung zwischen Keynesianern und Monetaristen in den sechziger und siebziger Jahren hat gezeigt, dass man sehr wohl eine gemeinsame Sprache zur Verfügung hatte, in der man die Kontroversen austragen konnte. Dies zeigt genauso wie auch z.B. jene Vexierbilder, die P.K. FEYERABEND (1976, S. 314; 1978, S. 193) zur Demonstration der Inkommensurabilität anführt, dass durch Kommunikation aufgezeigt werden kann, dass beide Perspektiven möglich sind und weshalb ich aus der einen zum Ergebnis A und aus der anderen zum Ergebnis B komme. ${ }^{58)}$

\section{Das wissenschaftliche Forschungsprogramm der Ökonomie}

[42] Das oben gezeichnete Bild des Paradigmas der Wirtschaftswissenschaften mag aus zwei Gründen kritisiert werden: Zum einen gibt es bereits seit einiger Zeit auch innerhalb der Disziplin erhebliche Kritik am ökonomischen Verhaltensmodell, insbesondere im Rahmen der

57. Zum ökonomischen Imperialismus siehe z.B. G. KIRCHGÄSSNER (2009a).

58. Siehe hierzu auch G. ANDERSSON (1988, S. 1121f.). Dies schliesst nicht aus, dass bestimmte Personen (aus welchen Gründen auch immer) nicht in der Lage sind, beide Perspektiven einzunehmen, aber es zeigt, dass man beide Perspektiven einnehmen kann, was die Behauptung der Inkommensurabilität widerlegt. 
,Behavioural Economics ${ }^{6}{ }^{59)}$ Andererseits wird dieses Modell häufig erheblich ,reicher' dargestellt, indem z.B. die Rationalitätsannahme sehr viel strenger ist und daneben die ebenfalls relativ starke Annahme der Eigeninteressiertheit menschlichen Handeln gesetzt wird. Im Extremfall haben wir das (häufig kritisierte) Modell des mikroökonomischen Einführungslehrbuchs, in welchem vollständig informierte Individuen ihren Nutzen dadurch maximieren, dass sie aus einem vorgegebenen Güterangebot zu festen Preisen das für sie ideale Güterbündel wählen.

[43] Beide Argumente sind richtig, aber sie treffen nicht den Kern des Ansatzes, der oben bewusst ,sparsam' dargestellt ist. Zur Beschreibung des Kerns bietet sich hier eher als der Ansatz TH.S. KUHN (1962) jener von I. LAKATOS (1974) an: In seiner Terminologie handelt es sich hier um die negative Heuristik, den harten Kern des Programms. Tatsächlich wird in der weit überwiegenden Anzahl ökonomischer Analysen sehr viel mehr unterstellt, und zwar sowohl bezüglich der Motivations- wie auch der Rationalitätsannahme, womit wir uns im Schutzgürtel des Programms befinden, um wiederum die Terminologie von I. LAKATOS (1974) zu verwenden. Dabei sind zwei Dinge offensichtlich, die auch niemanden überraschen sollten: Zum einen kann dieses Modell auch mit starken Annahmen in bestimmten Bereichen sehr viel erklären, zum anderen begibt man sich damit aber auch in ein Meer von Anomalien.

[44] All dies ist seit langem bekannt. Probleme mit der Rationalitätsannahme wurden zuerst zu Beginn der fünfziger Jahre im Rahmen der Diskussion des Allais-Paradoxes ${ }^{60)}$ und später vor allem im Rahmen der auf H.A. SIMON (1955) zurückgehenden Theorie eingeschränkter Rationalität thematisiert, Probleme der Eigennutzannahme wohl zuerst bei der Übertragung dieses Ansatzes auf den Bereich der Politik, als A. Downs (1957) und insbesondere W.H. RIKER und P.C. ORDESHOOK (1968) feststellten, dass ein Modell, welches das Verhalten von Wähler als rationale, ihrem eigenen Interesse dienende Handlung erklären soll, scheitert: Es kommt zum Paradox des Nicht-Wählens: Würde man sich im eigenen Interesse und rational verhalten, dürfte sich kaum jemand an einer Wahl oder Abstimmung beteiligen. In diesem Fall hat man nicht von der Rationalitäts-, sondern von der Eigennutzannahme Abstand genommen.

[45] Es ist das Verdienst der Behavioural Economics, in den letzten Jahrzehnten diese Probleme näher untersucht und hier erhebliche Fortschritte erzielt zu haben. Im Schutzgürtel des ökonomischen Forschungsprogramms waren dabei erhebliche Änderungen erforderlich. Der Kern wurde dadurch jedoch zumindest bisher nicht angetastet: Auch die Behavioural Economics gehen von Individuen mit Intentionen aus, die unter beschränkter Information im Sinne des schwachen Rationalitätsprinzips handeln. Die gelegentlich auftretende (eher terminologische) Verwirrung ergibt sich vor allem dadurch, dass die Vertreter dieses Ansatzes das ökonomische Verhaltensmodell recht umfassend definieren, womit es dann im Widerspruch zu

59. Zur Übersicht über die Behavioural Economics siehe z.B. C.F. CAMERER und G. LOEWENSTEIN (2004) oder A.C. SANTOS (2009).

60. Siehe hierzu M. Allais (1953). 
den Ergebnissen ihrer (zumeist experimentellen) Forschung steht und sich damit plakative Aussagen wie ,dass der Homo Oeconomicus tot sei, rechtfertigen lassen. ${ }^{61)}$

[46] Inwieweit die Ergebnisse der Behavioural Economics dazu führen, dass die Wirtschaftswissenschaften und insbesondere die Volkswirtschaftslehre (wieder) stärker politikrelevant werden, muss vorerst offen bleiben. Es gibt einige Ansätze im Rahmen des ,sanften Paternalismus', die in Zusammenhang mit meritorischen Gütern neue Möglichkeiten für bewusste politische Entscheide eröffnen. ${ }^{62)}$ In der Krise des Jahres 2009, die u.a. durch Modelle der Risikoabschätzung hervorgerufen wurde, die auf den extrem starken Rationalitätsannahmen von J. von NEUMANN und O. MORGENSTERn (1948) basieren, wurde gelegentlich auf die Ergebnisse der Behavioural Economics als mögliche Alternative zu den traditionellen Verfahren der Risikoabschätzung verwiesen. ${ }^{63)}$ Konkrete Vorschläge in dieser Richtung wurden freilich bisher keine gemacht, ganz abgesehen davon, dass diese sich auch in der Praxis zu bewähren hätten. Insofern haben wir bisher im Wesentlichen Ergebnisse, die im Labor für die Mikroebene abgeleitet wurden und die zeigen, dass bestimmt strenge Annahmen, die üblicherweise bei ökonomischen Analysen gemacht werden, in bestimmten Situationen nicht gelten. Inwieweit dies auf die Makroebene zu übertragen ist bzw. welche wirtschaftspolitische Relevanz dies hat, ist bisher weitgehend offen. Dies gilt insbesondere dann, wenn in derartigen Experimenten Phänomene wie die Geldillusion oder Suchtverhalten simuliert werden sollen.

[47] Eine Infragestellung des Kerns der ökonomischen Verhaltensmodells könnte sich allenfalls durch die Neuroökonomik ergeben. ${ }^{64)}$ Inwieweit dies tatsächlich geschehen wird, ist derzeit freilich reichlich spekulativ. Man kann sich auch eine zusätzliche Bewährung des traditionellen Modells des Homo Oeconomicus vorstellen. In Experimenten wurde gezeigt, dass Individuen selbst dann bereit sind, andere zu bestrafen, die sich nicht an bestimmte Normen halten, auch wenn dies für den Bestrafenden Kosten verursacht. Dies wurde als Widerlegung der Eigennutzannahme interpretiert. Wenn heute von der Neuro-Ökonomik gezeigt werden kann, dass durch diese Bestrafung die gleichen Gegenden im Gehirn aktiviert werden wie bei anderen Nutzen stiftenden Aktivitäten, sind wir zumindest wieder nahe an der Annahme, dass die Menschen genau dies tun, was ihnen Nutzen stiftet. Aber während dies bisher eine (nahezu) tautologische Aussage war, hätten wir jetzt eine experimentelle Unterlegung. Die Ergebnisse der Experimente könnten dann mit der Eigennutzannahme vereinbar sein.

61. Wenn z.B. AXEL OKENFELS, einer der prominenten jüngeren Vertreter der Behavioural Economics im deutschsprachigen Raum, den „Abschied vom Homo Oeconomicus“ einläuten will, geht es auch ihm lediglich um eine Modifikation der in ökonomischen Analysen üblicherweise gemachten Annahme der Verfolgung des Eigeninteresses, nicht um eine grundsätzliche Ablehnung des gesamten Ansatzes.

62. Zum sanften Paternalismus siehe z.B. G. KIRCHGÄSSNER (1991, S. 261ff.)

63. Siehe z.B. R. THALER, Die Finanzkrise ist kein Unfall, Institutional Money 1/2009, http://www. institutional-money.com/cms/magazin/theorie-praxis/artikel/die-finanzkrise-ist-kein-unfall/?tx_ttnews\%5Bpointer\% 5D=6\&tx_ttnews\%5BbackPid\%5D=18\&cHash=202b428285 (12/02/11).

64. Zur Neuroökonomik siehe z.B. G. Bonano, C. List, B. Tungodden und P. VAlentyne (2088), K.A. MCCABE (2008), G.,W. HARRISON (2088) sowie die (übrigen) Beiträge in Economics and Philosophy 24 (2008), Heft 3 und die Beiträge in Analyse und Kritik 29 (2007, Heft 1). 


\section{7 Ökonomische Normalwissenschaft und die Lösung von Rätseln}

[48] Soweit Ökonomen die Ideen von TH. KUHN (1962) aufgenommen haben, sei es, dass sie seine Konzepte auf die Entwicklung der ökonomischen Theorie angewendet haben, sei es, dass sie grundsätzliche Kritik geübt und die Anwendbarkeit seiner Konzeption in Frage gestellt haben, bezogen sie sich fast ausschliesslich auf seine Konzeptionen des Paradigmas und der wissenschaftlichen Revolutionen, aber kaum jemals auf seine Konzeption der Normalwissenschaft. ${ }^{65)}$ Diese scheint nicht nur bei Philosophen und Wissenschaftstheoretikern wenig Kredit zu geniessen, wie die Diskussionen der sechziger und siebziger Jahre zeigen, sondern auch bei den Ökonomen. Dabei scheint gerade das Konzept der Normalwissenschaft auf grosse Teile der heutigen wirtschaftswissenschaftlichen Forschung zu passen, ${ }^{66)}$ und genauso wie in den Naturwissenschaften ist dies zumindest teilweise ein notwendinger Bestandteil wissenschaftlicher Forschung, der bei Weitem nicht immer nur negativ zu bewerten ist. Man denke (im Bereich der Naturwissenschaften) nur an die vielen Wissenschaftler, die an den Grossexperimenten am CERN oder am DESY beteiligt sind.

[49] Einer der wenigen, die darauf aufmerksam gemacht haben, dass sich wirtschaftswissenschaftliche Forschung in aller Regel als Normalwissenschaft im Sinne von TH. KUHN (1962) vollzieht, ist J. WEIMANN (1984), wobei auch er dies als negativ ansieht. Nach ihm dürften jedem Ökonomen ,die typischen Symptome einer solchen Strategie bekannt sein: das beharrliche Festhalten an Theorien, deren Gültigkeit nur für eine idealtypische Modellwelt nachzuweisen ist; die Immunisierung von Theorien, entweder a priori durch explizite Bezugnahme auf einen rein theoretischen Begründungszusammenhang oder ad hoc durch die nachträgliche Einführung von Prämissen und Annahmen, die die ,wenn'-Komponente der theoretischen Aussage so aufblähen, dass die ,dann'-Komponente zum irrealen Spezialfall wird.“ (S. 284.) Er sieht den Grund dafür darin, dass die sozialwissenschaftliche (und damit auch die wirtschaftswissenschaftliche) Forschung im Wesentlichen von aussen bestimmt ist, ,ihre Rationalität weitgehend aus ihrer Fremdbestimmung bezieht" und sieht als Voraussetzung einer „Überwindung“ dieser Normalwissenschaft eine „Anerkennung der Wertigkeit sozialwissenschaftlicher Theorien“, sodass die „Zwecksetzung der Forschung, so sie unvermeidlich ist, zu einem demokratischen Prozess gedeihen kann, indem der Sozialwissenschaftler selbst eine bewusste, eine wichtige Rolle spielt.“ (S. 297.) Auch wenn man seine Analyse bezüglich der von ihm angeführten Gründe nicht teilt, wird man kaum umhinkommen, zugeben zu müssen, dass seine Beschreibung der wirtschaftswissenschaftlichen Forschung die Realität dieser Forschung zumindest nicht vollständig verfehlt.

[50] Will man der heutigen Situation der Normalwissenschaft in den Wirtschaftswissenschaften gerecht werden, sollte man zwischen der rein theoretischen und der empirischen Forschung unterscheiden. In der empirischen Forschung, auf die oben bereits eingegangen wurde, haben wir sowohl bei der Feld- wie bei der experimentellen Forschung heute die Situation,

65. Siehe hierzu die Übersicht in S.A. DraKopoulos und A. KARAYANNIS (2005).

66. Siehe hierzu auch V.J. TARASCIO (1997, S. 16): „Vieles, woraus heute Forschung und Publikationen in ökonomischen Zeitschriften besteht, kann als ,Normalwissenschaft' im Kuhn'schen Sinne dieses Begriffs charakterisiert werden: die Verfeinerung, sorgfältige Ausarbeitung und Anwendung von Theorien innerhalb eines bestehenden Paradigmas." 
dass im Rahmen grösserer Forschungsprojekte ähnlich wie in den Naturwissenschaften bestimmte Fragestellungen aufgeteilt und von einer Vielzahl von Doktorierenden bearbeitet werden. Als Beispiel mag die moderne mikroökonometrische Arbeitsmarktforschung dienen. Wie oben bereits ausgeführt wurde, wurden im Bereich der Mikroökonometrie durch und im Anschluss an Arbeiten von JAMES J. HECKMANN Methoden entwickelt, die im Vergleich zu den früher angewendeten Verfahren z.B. sehr viel zuverlässigere Aussagen darüber erlauben, ob bestimmte Massnahmen der aktiven Arbeitsmarktpolitik tatsächlich dazu führen, dass die Teilnehmerinnen und Teilnehmen an diesen Massnahmen schneller wieder einen Arbeitsplatz finden. ${ }^{67)}$ Diese Methoden wurden und werden allein im deutschsprachigen Raum heute an verschiedenen Universitäten und in mehreren Wirtschaftsforschungsinstituten auf unterschiedliche Perioden und Länder angewendet, wobei sie teilweise auch an die dortigen Situationen angepasst werden müssen, woraus z.B. eine ganze Reihe von Dissertationen entstanden ist bzw. noch entsteht. ${ }^{68)}$ Dies ist ohne Frage Normalwissenschaft; der methodologische Rahmen ist sowohl von der theoretischen als auch von der statistischen Seite her vorgegeben und wird nicht in Frage gestellt. Die Wissenschaftler lösen ,Puzzles' im Sinne von TH.S. KuHN (1962). Freilich ist diese Forschung alles andere als trivial, und sie ist zudem gesellschaftlich ausserordentlich wichtig: Es geht um viele Milliarden Euro (bzw. CHF), die, wie die Ergebnisse dieser Forschung zeigen, häufig sinnlos ausgegeben werden, d.h. ohne dass sie die erhofften positiven Auswirkungen auf die Beschäftigung auslösen. ${ }^{69)}$

[51] In einer ähnlichen Situation befinden sich grosse Teile (wenn nicht sogar der weitaus grösste Teil) der empirischen Wirtschaftswissenschaften (wie auch anderer empirischer Sozialwissenschaften): Es geht zwar um die Überprüfung einzelner Hypothesen und damit um die Aufarbeitung von Fakten, aber kaum um die Überprüfung bzw. Verwerfung ganzer Theorien. Dabei können solche Untersuchungen nicht nur einen erheblichen Schwierigkeitsgrad aufweisen, sondern auch diese Ergebnisse sind immer nur ,vorläufig', da dahinter Messtheorien stehen, die selbst fehlerhaft sein können, so dass sie zu falschen Ergebnissen führen. Schliesslich hat bereits K. R. POPPER (1935, S. 60ff.) darauf hingewiesen, dass es keine theoriefreie Beobachtung gibt, und I. LAKATOS (1974) sowie P.K. FEYERABEND (1976) haben viele ihrer Argumente in der Diskussion Anfang der siebziger Jahre darauf aufgebaut. Zudem hat diese Forschung trotz aller ihrer gesellschaftlichen Relevanz den Nachteil, dass sie oft schon nach wenigen Jahren bestenfalls Wirtschaftshistoriker (und vielleicht noch jene, die sich mit Dogmengeschichte befassen,) interessiert: Die korrekte Spezifikation einer Geldnachfragefunktion für Deutschland in den achtziger und neunziger Jahren des letzten Jahrhunderts interessiert heute bestenfalls noch im Vergleich mit der heutigen Geldnachfragefunktion im Euro-Raum, so wichtig sie damals für die Politik der Deutschen Bundesbank auch war.

67. Siehe hierzu z.B. M. LECHNER und F. PFEIFFER (2001).

68. Allein in Deutschland haben neben dem Institut für die Zukunft der Arbeit (IZA) in Bonn z.B. das Zentrum für Europäische Wirtschaftsforschung (ZEW) in Mannheim sowie das Rheinisch-Westfälische Institut für Wirtschaftsforschung (RWI) in Essen einen Schwerpunkt im Bereich der mikroökonometrischen Arbeitsmarktforschung.

69. Siehe hierzu z.B. M. LECHNER und C. WunSCH (2008). 
[52] Bei vielen besteht die Hoffnung, dass dies im Bereich der theoretischen, heute in aller Regel stark mathematisch ausgerichteten Forschung zumindest partiell anders ist. Aber auch dort kann es geschehen, dass das Problem, welches analysiert wird, in der praktischen Wirtschaftspolitik zwar heute relevant ist, aber in zehn oder zwanzig Jahren, aus welchen Gründen auch immer, irrelevant sein wird. Dennoch besteht die Hoffnung, dass ein theoretisches Ergebnis, wenn es einmal erzielt ist, auf Dauer gültig sein wird. Davon kann man auch deshalb ausgehen, weil eine mathematische Ableitung bzw. ein Beweis, wenn diese bzw. dieser gelungen ist, allein durch den Zeitablauf nicht falsch oder irrelevant wird; schlimmstenfalls wird ein Beweis gefunden, der (noch) einfacher bzw. eleganter ist und den alten Beweis dadurch überflüssig macht. Wie das Beispiel des Allgemeinen Unmöglichkeitsthorems von K.J. ARROW (1951) zeigt, muss jedoch weder ein kleiner Fehler in der Beweisführung noch die Tatsache, dass später einfachere Beweise gefunden wurden, dazu führen, dass die ursprüngliche Leistung in Vergessenheit gerät; sie kann sogar mit einem Nobelpreis belohnt werden.

[53] Diese Arbeit ist jedoch ein (extremer) Ausnahmefall und in keiner Weise repräsentativ für den Grossteil der heute produzierten Ergebnisse in der rein mathematisch-theoretischen Forschung. Zum einen geht es gelegentlich nur darum, mathematisch-elegante Formulierungen für eher triviale Ergebnisse zu finden, wie z.B. für die Tatsache, dass Menschen umso eher ihrem eigenen Leben ein Ende setzen, je weniger sie für sich von der Zukunft erwarten. ${ }^{70)}$ Entscheidender ist, dass viele dieser Ergebnisse ausser den Autoren (und möglicherweise noch einer kleinen Gruppe von Forschern) kaum jemanden interessieren; sie werden kaum gelesen und dementsprechend auch nicht zitiert. Es gab vor einigen Jahren in Deutschland ein Experiment, welches aus rechtlichen Gründen jedoch (leider) abgebrochen werden musste, bevor die endgültigen Ergebnisse vorlagen. Bei verschiedenen wirtschaftswissenschaftlichen Fachzeitschriften wurden Aufsätze zum zweiten Mal eingereicht, die dort bereits publiziert worden waren. Verändert wurden (neben dem Datum) nur der Autor und der Titel der Arbeit. Als das Experiment abgebrochen werden musste, waren einige Aufsätze bereits in der gleichen Zeitschrift zum zweiten Mal akzeptiert. Diese Arbeiten waren insofern qualitativ gut, als sie zum zweiten Mal den Begutachtungsprozess überstanden haben, aber ihre Ergebnisse waren so irrelevant, dass weder die Herausgeber noch die Gutachter, die ja eigentlich Spezialisten auf dem jeweiligen Gebiet sein sollten, wussten bzw. bemerkten, dass diese Arbeiten bereits in dieser Zeitschrift publiziert waren. ${ }^{71)}$

[54] Damit stellt sich die Frage, was der Sinn solcher Arbeiten ist. Selbstverständlich kann nie ausgeschlossen werden, dass in einer solchen Arbeit gelegentlich ein bahnbrechendes Ergebnis erzielt wird, welches nicht nur der Karriere des Forschers zuträglich ist, sondern auch die Wissenschaft voranbringt. Derartige Ausnahmefälle können freilich kaum das ganze Unternehmen rechtfertigen; schliesslich werden hier in erheblichem Masse gesellschaftliche Ressourcen eingesetzt, für die es auch alternative Verwendungen gäbe. Auch wenn man der Wis-

70. Zur ökonomischen Theorie des Selbstmords siehe D.S. HAMERMESH und N.M. Soss (1974), zur Kritik daran z.B. M. PRISCHING (1983).

71. In die gleiche Richtung gehen aufgedeckte Betrugsfälle, in denen Arbeiten anderer Autoren unter Abänderung des Namens von einem anderen Autor bei einer anderen Zeitschrift eingereicht und dort auch akzeptiert wurden. 
senschaft - mit guten Gründen - weitestgehend die Freiheit lassen will, über ihre Fragestellungen und Methoden selbst zu entscheiden, ist das Ausmass, in welchem Umfang gesellschaftliche Ressourcen dazu eingesetzt werden sollen, ein politischer Entscheid, der auch politisch gerechtfertigt werden muss. Dabei ist der Hinweis auf die gesellschaftlichen Vorteile der Wissenschaftsfreiheit und der Förderung der Wissenschaft für sich allein betrachtet nicht ausreichend. ${ }^{72)}$

[55] Die wesentliche Funktion, welche diese kaum gelesenen Beiträge haben, ist, dass die Autoren damit ihre Fähigkeit zeigen, derartige Rätsel zu lösen, was immer sie letztlich auch seien. Es geht somit weniger um wissenschaftlichen Fortschritt, als vielmehr um den Ausweis bestimmter Qualifikationen. ${ }^{73)}$ Was früher die Funktion der Habilitation war, ist heute gleichsam an Peer Groups delegiert, die über die Erlaubnis zur Veröffentlichung in wissenschaftlichen Zeitschriften entscheiden. Dies ist nicht notwendigerweise schlecht, aber man muss die Frage stellen, inwieweit der mit diesen Verfahren ursprünglich intendierte Nebeneffekt, dass dadurch relevante Forschungsergebnisse erzielt werden, im Bereich der Wirtschaftswissenschaften heute (noch) gegeben ist. ${ }^{74)}$

[56] Dies war vermutlich einer der rationalen Hintergründe, die hinter dem oben erwähnten, im Jahr 2009 von 83 (meist älteren) Professoren der Volkswirtschaftslehre unterschriebenen Aufruf „Rettet die Wirtschaftspolitik an den Universitäten“ lag. ${ }^{75)}$ Dass in diesem Zusammenhang gegen die mathematische Ausrichtung weiter Teile der heutigen Wirtschaftswissenschaften polemisiert wurde, mag mit dieser Funktion erheblicher Teile dieser Wissenschaft (und damit dem Funktionsverlust bezüglich der Generierung gesellschaftlich relevanter wissenschaftlich abgesicherter Aussagen) zusammenhängen. Auch wenn man über die Rolle der Mathematik in den Wirtschaftswissenschaften sowie insbesondere im Rahmen der wirtschaftswissenschaftlichen Grundausbildung diskutieren und dabei unterschiedlicher Meinung sein kann, greift man zu kurz, wenn man die Mathematisierung für die Entwicklung in der modernen, theoretisch ausgerichteten Volkswirtschaftslehre verantwortlich machen will und deshalb deren Zurückdrängung verlangt, da damit dieser grundlegende Funktionswandel dieser Art von Wissenschaft nicht beeinflusst wird.

\section{Zusammenfassung und abschliessende Bemerkungen}

[57] Ob wir dies schätzen oder nicht: Die meisten Forschungsaktivitäten in den Wirtschaftswissenschaften fallen in Kuhn-scher Terminologie heute in den Bereich der Normalwissenschaft. Wissenschaftliche Revolutionen sind, wenn man diesen Terminus ernst nimmt, ausgesprochen selten; sie haben in den vergangenen Jahrhunderten vielleicht zweimal

72. Siehe hierzu auch G. KIRCHGÄSSNER (2007).

73. In diesem Zusammenhang dürfte es nicht rein zufällig sein, dass sich W. THOMSON (1999) in der im Journal of Economic Literature und damit an sehr prominenter Stelle veröffentlichten Anweisung, wie junge Ökonomen Arbeiten im Bereich der ökonomischen Theorie Papiere verfassen sollten, auf rein formale Hinweise beschränkt; die gesellschaftliche und/oder wirtschaftspolitische Relevanz ist dabei kein Thema.

74. Zu dieser Selektionsfunktion siehe auch B.S. FREY (2000, S. 26).

75. Siehe http://www.faz.net/s/RubB8DFB31915A443D98590B0D538FC0BEC/Doc EA1E6687105BC44399 168BC77ADE64F8A ATpl Ecommon Scontent.html (01.02.11). 
stattgefunden. Das grundlegende Paradigma der Wirtschaftswissenschaften bzw. der harte Kern ihres Forschungsprogramms ist weitgehend unbestritten, auch wenn über den Schutzgürtel heftig gestritten wird und hier gerade aus der interdisziplinären Zusammenarbeit mit den Psychologen Fortschritte erzielt wurden.

[58] Kaum bestreitbare Fortschritte gab es auch im Bereich der empirischen Verfahren sowie ihrer Anwendung auf wirtschaftliche Fragstellungen. Aber auch hier befinden wir uns im Bereich der Normalwissenschaft, auch wenn die dort erzielten Ergebnisse teilweise nicht nur wissenschaftlich interessant, sondern auch für die praktische Wirtschaftspolitik von hoher Relevanz sind. Behauptungen, der derzeitige Weg einer empirisch-quantitativen Forschung sei ein Irrweg, man müsse sich von dieser von den Angelsachsen dominierten Entwicklung (weitgehend) abkoppeln und zurück zur (deutschen) Ordnungstheorie finden, wie sie in Deutschland in der Debatte des Jahres 2009 zu hören waren, sind eigentlich nur vor dem Hintergrund bestimmter (enttäuschter) politischer Vorstellungen verständlich, ganz abgesehen davon, dass die Intentionen der Ordnungsökonomik, nicht nur die wirtschaftlichen Prozesse selbst zu analysieren, sondern auch deren institutionelle Rahmenbedingen in die Analyse einzubeziehen, gerade auch durch die moderne Institutionenökonomik aufgegriffen wurden, wobei kein Anlass bestand und besteht, dabei auf das moderne Instrumentarium der Ökonomik zu verzichten.

[59] Dass sich andererseits im Bereich der Wirtschaftswissenschaften eine Kultur der Rätsellösung entwickelt hat, wobei die Rätsel häufig wenig Bezug zu wirtschaftspolitischen Fragestellungen haben, lässt sich kaum bestreiten. Dies bedeutet jedoch weder, dass es in der wirtschaftswissenschaftlichen Forschung keinen Fortschritt gäbe, noch dass diese Forschung insgesamt keine wirtschaftspolitische Relevanz mehr besässe. Was den Fortschritt anbetrifft, sind wir freilich in keiner prinzipiell anderen Situation als andere Wissenschaften: Auch dort ist nicht a priori klar, dass neue theoretische Ansätze wirklich erklärungskräftiger sind als die bereits bekannten. Dies kann jedoch kein Grund dafür sein, nicht nach neuen Ansätzen zu suchen. Die (zumindest teilweise) in die gegenteilige Richtung zielende Aufforderung, sich eher auf die Vermittlung grosser Botschaften als auf sorgfältige theoretische Arbeit zu konzentrieren, wie sie von H. SIEBERT (1998) ergangen ist, kann man zumindest aus kritisch-rationaler Perspektive nicht unterstützen. ${ }^{76}$

76. „Das Fach muss stärker daran denken, dass es Verantwortung in der Gesellschaft hat und dass auch diejenigen von der großen Konzeption der Ökonomie zu überzeugen sind, die außerhalb des Fachs stehen, die in den Medien vermitteln müssen und die in der Politik zu entscheiden haben. ... Vielleicht sollten wir doch nicht jeden einzelnen Stein in der Wüste von Kakutania umdrehen, um danach zu suchen, wo der Wettbewerb nicht funktioniert, ... Und vielleicht, sollten wir alle einen Tick stärker berücksichtigen, was die Volkswirtschaftslehre insgesamt an verlässlichen Erkenntnissen zu bieten hat: Lassen Sie mich deshalb doch lieber ... mit einem Klassiker beschliessen, mit John Stuart Mill: ,If competition hat its evils, it prevents greater evils ... “ “ H. SIEBERT (1998, S. 62f.). 


\section{Zusammenfassung}

Zuerst wird auf die Positionen verschiedener Ökonomen zu Falsifikationismus, wissenschaftliche Revolutionen und wissenschaftliche Forschungsprogramme eingegangen. Danach wird der kaum bestreitbare Fortschritt dargestellt, der sich in den letzten Jahrzehnen im methodisch-empirischen Bereich vollzogen hat. Im Anschluss daran wird gefragt, ob es neben diesem Fortschritt, der sich vergleichsweise kontinuierlich vollzieht, auch Revolutionen gegeben hat, die sich insbesondere im Bereich der Theorie (bzw. der grundlegenden Methodologie) vollzogen haben. Anschliessend beschäftigen wir uns mit dem Paradigma, welches hinter dem ökonomischen Ansatz steht, sowie mit dem entsprechenden Forschungsprogramm und der Kritik daran. Im Anschluss daran werden Probleme der Normalwissenschaft in den Wirtschaftswissenschaften diskutiert, die heute ohne Zweifel den grössten Raum im Rahmen der wirtschaftswissenschaftlichen Forschung einnimmt. Auch wenn damit einige Probleme verbunden sind, besteht kein Anlass, diese Forschung in irgendeiner Weise abzuwerten.

\section{Literaturangaben}

D. Acemoglu (2009), The Crisis of 2008: Structural Lessons For and From Economics, Centre for Economic Policy Research Policy Insight No. 28, London, January 2009. (http://www.cepr. org/pubs/PolicyInsights/PolicyInsight28.pdf (11/02/11))

H. Albert (1963), Modell-Platonismus: Der neoklassische Stil des ökonomischen Denkens in kritischer Beleuchtung, in: F. KARRENBERG und H. ALBERT (eds.), Sozialwissenschaft und Gesellschaftsgestaltung, Duncker und Humblot, Berlin 1963, S. 45 - 76; abgedruckt in: H. ALBERT Marktsoziologie und Entscheidungslogik, Luchterhand, Neuwied/Berlin 1967, S. 331 - 367. (Neuauflage: Mohr (Siebeck), Tübingen 1998.)

H. ALBERT (1978), Nationalökonomie als sozialwissenschaftliches Erkenntnisprogramm, in: H. ALBERT u.a., Ökonometrische Modelle und Sozialwissenschaftliche Erkenntnisprogramme, Bibliographisches Institut - Wissenschaftsverlag, Zürich 1978, S. 49 - 71.

H. AlBeRT (1986), Anmerkungen zum ökonomischen Denken, Konstanzer Blätter für Hochschulfragen 23 (1986), S. 6 - 17.

H. AlBerT (1990), Der Mythos des Rahmens am Pranger: Anderssons Antwort auf die wissenschaftsgeschichtliche Herausforderung, Zeitschrift für philosophische Forschung 44 (1990), S. 85 - 97.

H. Albert (2006), Die ökonomische Tradition und die Verfassung der Wissenschaft, Perspektiven der Wirtschaftspolitik 7 (2006), Special Issue, S. 113 - 131.

M. Allais (1953), Le comportement de l'homme rationnel devant risque: Critique des postulats et axiomes de l'école Américaine, Econometrica 21 (1953), pp. 503 - 546; extended english version: The Foundations of a Positive Theory of Choice Involving Risk and a Criticism of the Postulate and Axioms of the American School, in: M. Allais and O. HAGEN (eds.), Expected Utility Hypotheses and the Allais Paradox, Reidel, Dordrecht 1979, pp. 27 - 145.

G. ANDERSSON (1988), Kritik und Wissenschaftsgeschichte: Kuhns, Lakatos' und Feyerabends Kritik des Kritischen Rationalismus, Mohr (Siebeck), Tübingen 1988.

K.J. Arrow (1951), Social Choice and Individual Values, Wiley, New York 1951. (2. Auflage 1963.)

R.E. BACKHOUSE (1997), Truth and Progress in Economic Knowledge, Edward Elgar, Cheltenham und Lyme 1997. 
R.E. BACKhouse (2004), The Methodology of Scientific Research Programmes, in: J.B. DAVIS, A. MARCIANO und J. RUNDE (eds.), The Elgar Companion to Economics and Philosophy, Edward Elgar, Cheltenham 2004, S. 181 - 196.

R.E. BALDwIN (1992), Are Economist's Traditional Trade Policy Views Still Valid?, Journal of Economic Literature 30 (1992), S. 804 - 829.

H.M. BLALOcK (ed.) (1971), Causal Models in the Social Sciences, Macmillan, London und Basingstoke 1971.

M. BlAUG (1976), Kuhn versus Lakatos or Paradigms versus Research Programmes in the History of Economics, in: S. LATSIS (1976, S. 149 - 180); gekürzt abgedruckt in und zitiert nach D.M. HAUSMAN (ed.), The Philosophy of Economics: An Anthology, Cambridge University Press, Cambridge (UK), 2. Auflage 1994, S. 348 - 375.

M. Blaug (1980), The Methodology of Economics: Or How Economists Explain, Cambridge University Press, Cambridge (U.K.) et al., 2. Auflage 1992.

M. Blaug (1990), Second Thoughts on the Keynesian Revolution, in: M. BlaUg, Economic Theories: True or False?, Edward Elgar, Aldershot 1990, S. 88 - 106; abgedruckt in: B.J. CALDWELL (1993).

G. BonAno, C. List, B. TUngodden und P. VALENTYNe (2088), Introduction to the Special Issue of Economics and Philosophy on Neuroeconomics, Economics and Philosophy 24 (2008), S. 301 302.

A. BÖRSCH SUPAN und H. JÜRgeS (2005) (eds.), The Survey on Health, Ageing and Retirement in Europe: Methodology, Mannheim Research Institute on the Economics of Ageing (MEA), Mannheim 2005. (http://www.share-project.org/t3/share/index.php?id=books\&no_cache=1.)

A. BÖRSCH SUPAN et al. (2005) (eds.), Health, Ageing and Retirement in Europe: First Results from the Survey on Health, Ageing and Retirement in Europe, Mannheim Research Institute on the Economics of Ageing (MEA), Mannheim 2005. (http://www.share-project.org/t3/share/ index.php?id=books\&no_cache $=1$.)

M. BRONFENBRENNER (1971), The Structure of Revolutions in Economic Thought, History of Political Economy 3 (1971), S. 136 - 151.

W.H. Buiter, (1980), The Macroeconomics of Dr. Pangloss, A Critical Survey of the New Classical Macroeconomics, Economic Journal 90 (1980), S. 34 - 50.

B.J. CALDwell (1991), Clarifying Popper, Journal of Economic Literature 29 (1991), S. 1 - 33.

B.J. CALDwell (1993) (ed.), The Philosophy and Methodology of Economics, Band III, Edward Elgar, Aldershot 1993.

C.F. CAMERer und G. Loewenstein (2004), Behavioral Economics: Past, Present, Future, in: C.F. CAMERer, G. LoewensteIn und M. RABIN (2004), Advances in Behavioral Economics, Princeton University Press, Princeton/Oxford 2004, S. 3 - 51.

A.C. SANTOS (2009), Behavioral Economics: How and What Can We Learn About Human Behavior, Journal of Economic Methodology 16 (2009), S. 71 - 88.

G.C. ChOw und S.B. MegDAL, (1978), An Econometric Definition of the Inflation-Unemployment Trade-off, American Economic Review 68 (1978), S. 446 - 453.

T. COUPÉ (2004), What Do We Know about Ourselves? On the Economics of Economics, Kyklos 57 (2004), S. $197-216$.

A. Downs (1957), An Economic Theory of Democracy, Harper and Row, New York 1957; deutsche Übersetzung: Ökonomische Theorie der Demokratie, Mohr (Siebeck), Tübingen 1968.

S.A. DRAKopoulos und A. KARAYANNIS (2005), A Review of Kuhnian and Lakatosian ,Explanations“ in Economics, MPRA Working Paper Nr. 16624, Januar 2005. 
E. DuRKheIM (1895), Les règles de la méthode sociologique, Presses Universitaires de France, Paris 1895; deutsche Übersetzung: Die Regeln der soziologischen Methode, Luchterhand, Neuwied 1961.

T. EGGERTSSON (1995), On the Economics of Economics, Kyklos 48 (1995), S. $201-210$.

H. ESSER (1993), The Rationality of Everyday Behavior : A Rational Choice Reconstruction of the Theory of Action by Alfred Schütz, Rationality and Society 5 (1993), S. 7 - 31.

O. FABel, M. HeIN und R. HofmeISTER (2008), Research Productivity in Business Economics: An Investigation of Austrian, German and Swiss Universities, German Economic Review 9 (2008), S. $506-531$.

P.K. FEYERABEND (1976), Wider den Methodenzwang, Skizze einer anarchistischen Erkenntnistheorie, Suhrkamp, Frankfurt 1976.

P.K. FEYERABEND (1978), Kuhns Struktur wissenschaftlicher Revolutionen: Ein Trostbüchlein für Spezialisten, in: P.K. FEYERABEND, Der wissenschaftstheoretische Realismus und die Autorität der Wissenschaften, Vieweg, Braunschweig/Wiesbaden 1978, S. 153 - 204.

M. Friedman (1948), A Monetary and Fiscal Framework for Economic Stability, American Economic Review 38 (1948), S. 245 - 264.

B.S. FreY (2000), Was bewirkt die Volkswirtschaftslehre?, Perspektiven der Wirtschaftspolitik 1 (2000), S. $5-33$.

B.S. FreY (2005), Problems With Publishing: Existing States and Solutions, European Journal of Law and Economics 19 (2005), S. 173 - 190.

B.S. FREY (2010). Withering Academia, CESifo Working Paper Nr. 3209, München, Oktober 2010.

M. Friedman (1953), The Methodology of Positive Economics, in M. Friedman, Essays in Positive Economics, University of Chicago Press, Chicago 1953, S. 3 - 43.

M. FriedMAn (1968), The Role of Monetary Policy, American Economic Review 58 (1968), S. 117.

M. Friedman und A.J. Schwarz (1965), A Monetary History of the United States, 1867-1960, Princeton University Press, Princeton 1965.

Ch.L. GiLbert (1991), Do Economists Test Theories?: Demand Analysis and Consumption Analysis as Tests of Theories of Economic Methodology, in: N. DE MARCHI und BlAUG (1991), S. 137 168.

R.J. GORDON (1990), What is New-Keynesian Economics? Journal of Economic Literature 28 (1990), S. $1115-1171$.

T. GOSPODAREK (2009), 'Representative management' as a rational research program in Kuhn-Lakatos-Laudan sense, International Journal of Economics and Business Research 1 (2009), S. 409 421.

M. Graber, A. LaunOV und K. WÄLde (2008). Publish or Perish? The Increasing Importance of Publications for Prospective Economics Professors in Austria, Germany and Switzerland, German Economic Review 9 (2008), S. 457 - 472.

C.W.J. GRANGER und P. NEWBOLD (1975), Economic Forecasting: The Atheist's Viewpoint, In: G.A. RENTON (ed.): Modelling the Economy. Heineman, London 1975, S. 131 - 148.

D.S. HAMERMESH und N.M. SOSS (1974), An Economic Theory of Suicide, Journal of Political Economy 82 (1974), S. 83-98.

D.W. HAND (1985), Second Thoughts on Lakatos, History of Political Economy 17 (1985), S. 1 16; abgedruckt in: B.J. CALDWELL (1993). 
G.,W. HARRISON (2008), Neuroeconomics: A Critical Reconsideration, Economics and Philosophy 24 (2008), S. $303-344$.

D. Hausman (1989), Economic Methodology in a Nutshell, Journal of Economic Perspectives 3 (1989), Heft 2, S. $115-127$.

J. HICKS (1976), 'Revolutions' in Economics, in S. LATSIS (1976), S. 207 - 218.

F. Hoffmann und P. ORepoulos (2009), Professor Quality and Student Achievement, Review of Economics and Statistics 91(2009), S. 83 - 92.

D. Hume (1752), Political Discourses, Kinkaid und Donaldson, Edinburgh 1752; deutsche Übersetzung enthalten in: Politische und ökonomische Essays, Meiner, Hamburg 1988.

R. IngLeHART et al. (2004), Human Beliefs and Values: A Cross-Cultural Sourcebook Based on the 1999 - 2002 Values Surveys. Siglo XXI Editores, Mexico 2004.

J.M. KEYNES (1936), The General Theory of Employment, Interest, and Money, Macmillan, London 1936; deutsche Übersetzung: Allgemeine Theorie der Beschäftigung, des Zinses und des Geldes, München 1936.

J. KIM (1991), Testing in Modern Economics: The case of Job Search Theory, in: N. DE MARCHI und BLAUG (1991), S. $105-131$.

G. KIRCHGÄSSNER (1991), Homo Oeconomicus, Das ökonomische Modell individuellen Verhaltens und seine Anwendung in den Wirtschafts- und Sozialwissenschaften, J.B.C. Mohr (Paul Siebeck), Tübingen 1991; 3. Auflage 2008.

G. KIRCHGÄSSNER (2004), The Weak Rationality Principle in Economics, Volkswirtschaftliche Abteilung der Universität St. Gallen, Diskussionspapier Nr. 2004-13, Dezember 2004.

G. KIRCHGÄSSNER (2007), Wissenschaft und Politik: Chancen und Gefahren einer unvermeidlichen Allianz, in: W. BERKA und H. SCHMIDINGER (eds.), Vom Nutzen der Wissenschaften, Böhlau, Wien et al. 2007 , S. $183-219$.

G. KIRCHGÄSSNER (2009), Die Krise der Wirtschaft: Auch eine Krise der Wirtschaftswissenschaften?, Perspektiven der Wirtschaftspolitik 10 (2009), S. 436 - 468.

G. KIRCHGÄSSNER (2009a), Ökonomie: Die imperialistische Sozialwissenschaft, in: P. RUSTERHOLZ, R. MEYER SCHWEIZER und S.M. ZWAHLEN (eds.), Aktualität und Vergänglichkeit der Leitwissenschaften, Peter Lang, Bern et al. 2009, S. 59 - 95.

J. Kornai (1971), Anti-Equilibrium: On Economic Systems Theory and the Task of Research, NorthHolland, Amsterdam/London 1971.

P. Krugman (1987), Is Free Trade Passé?, Journal of Economic Perspectives 1 (1987), Heft 2, S. 131 $-144$.

TH. S. KuHN (1962), The Structure of Scientific Revolutions, The University of Chicago Press, Chicago 1962; deutsche Übersetzung: Die Struktur wissenschaftlicher Revolutionen, Suhrkamp, Frankfurt, 2. Auflage 1976.

TH. S. KuHN (1974), Logik der Forschung oder Psychologie der wissenschaftlichen Arbeit, in: I. LAKATOS und A. MuSGRAVE (1974), S. 1 - 24.

I. LAKATOS (1974), Falsifikation und die Methodologie wissenschaftlicher Forschungsprogramme, in: I. LAKATOS und A. MuSGRAVE (1974), S. 89 - 189.

I. LaKatos (1976), Proofs and Refutations: The Logic of Mathematical Discovery, Cambridge University Press, London et al. 1976.

I. LAKATOS und A. MusgRAVE (eds.) (1974), Kritik und Erkenntnisfortschritt, Vieweg, Braunschweig 1974. 
S. LATSIS (1972), Situational Determinism in Economics, British Journal for the Philosophy of Science 23 (1972), S. $207-245$.

S. LATsis (ed.) (1976), Method and Appraisal in Economics, Cambridge University Press, Cambridge 1976.

M. LeChNER und F. PFEIFFER (2001) (eds.), Econometric Evaluation of Labour Market Policies, Physica, Heidelberg 2001.

M. LECHNER und C. WunsCh (2008), What Did All The Money Do? On the General Ineffectiveness of Recent West German Labour Market Programmes, Kyklos 61 (2008), S. 134 - 174.

A. LEON (2006), The Effect of Education on Fertility: Evidence from Compulsory Schooling, University of Pittsburgh, Department of Economics, Working Paper Nr. 288, 2006.

A. LeJONHUfVUd (1976), Schools, 'Revolutions' and Research Programmes in Economic Theory, in S. LATSIS (1976), S. $65-108$.

F. MACHLUP (1960), Der Wettstreit zwischen Mikro- und Makrotheorien der Nationalökonomie, Mohr (Siebeck), Tübingen 1960.

U. MÄKI (1999), Science as a Free Market: A Reflexivity Test in an Economics of Economics, Perspectives on Science 7 (1999), S. 486 - 509.

U. MÄKI (2009) (ed.), The Methodology of Positive Economics: Reflections on the Milton Friedman Legacy, Cambridge University Press, Cambridge et al. 2009.

N.G. MANKIW (1990), A Quick Refresher Course in Macroeconomics. Journal of Economic Literature 28 (1990), S. 1645 - 1660.

N. DE MarChI und Blaug (eds.) (1991), Appraising Economic Theories: Studies in the Methodology of Research Programmes, Edward Elgar, Alderhot und Brookfield, 1991.

K.A. MCCABE (2008), Neuroeconomics and the Economic Sciences, Economics and Philosophy 24 (2008), S. $345-368$.

D. MCCloskey (1983), The Rhetoric of Economics, Journal of Economic Literature 21 (1983), S. $481-517$.

W. MeYer (1973b), Falsifikationslehre und ökonomische Theorie, Anwendungsprobleme des Kritischen Rationalismus, Wirtschaftswissenschaftliches Studium 2 (1973), S. 501 - 506; abgedruckt in: H. RAFFÉE und B. ABEL (eds.), Wissenschaftstheoretische Grundfragen der Wirtschaftswissenschaften, Vahlen, München 1979, S. 44 - 59.

L. V. Mises (1949), Human Action: A Treatise on Economics, Henry Regnery, Chicago, 3. Auflage 1966.

B. Mueller und St.C. Wolter (2011), The Consequences of being Different: Statistical Discrimination and the School-to-Work Transition, Leading House Economics of Education, Firm Behaviour, Training Policies, Working Paper Nr. 53, Zürich/Bern, Februar 2011.

H. MÜLLER-GODEFFroY (1985), Wissenschaftslogik und Wissenschaftsprozess: Zur Forderung nach Falsifizierbarkeit der Theorien der Nationalökonomie, Jahrbuch für Sozialwissenschaft 36 (1985), S. $128-148$.

J. Muth (1961), Rational Expectations and the Theory of Price Movements. Econometrica 29 (1961), S. $315-335$.

J. V. Neumann und O. Morgenstern (1944), Theory of Games and Economic Behavior, Princeton University Press, Princeton 1944; deutsche Übersetzung: Spieltheorie und wirtschaftliches Verhalten, Physica, Würzburg, 3. Auflage 1973.

N. OKISHIO (1963), A Mathematical Note on Marxian Theorems, Weltwirtschaftliches Archiv 91 (1963), S. $287-299$. 
A.W. PhILliPs (1958), The Relation Between Unemployment and the Rate of Change of Money Wage Rates in the United Kingdom, 1861 - 1957, Economica 25 (1958), S. 283 - 299.

Th. Polleit (2009), Mit Positivismus und Empirismus gegen die Freiheit, Ordnungspolitischer Blog ,Wirtschaftliche Freiheit", 8. August 2009. (http://wirtschaftlichefreiheit.de/wordpress/?p=1394 $(11 / 02 / 11)$.)

K.R. POPPER (1935), Logik der Forschung, Julius Springer, Wien 1935; 6. erweiterte Auflage, J.C.B. Mohr (Siebeck), Tübingen 1976.

K.R. POPPER (1962), Die Logik der Sozialwissenschaften, Kölner Zeitschrift für Soziologie und Sozialpsychologie 14 (1962), S. 233 - 248; abgedruckt in: TH.W. ADORNO et al., Der Positivismusstreit in der deutschen Soziologie, Luchterhand, Neuwied/Berlin 1969, S. 103 - 123.

K.R. POPPER (1963), Conjectures and Refutations: The Growth of Scientific Knowledge, Routledge and Kegan Paul, London, 4. Auflage 1972.

K.R. POPPER (1972), Objective Knowledge, Clarendon Press, Oxford, 1972; deutsche Übersetzung: Objektive Erkenntnis: Ein evolutionärer Entwurf, Hoffmann und Campe, Hamburg 1973.

K.R. POPPER (1983), Realism and the Aim of Science, From the Postscript to the Logic of Scientific Discovery, Hutchinson, London 1983; zitiert nach der deutschen Übersetzung: Realismus und das Ziel der Wissenschaft, Aus dem Postskript zur Logik der Forschung I, Mohr Siebeck, Tübingen 2002.

M. PRISCHING (1983), Über die Karriere einer Handlungstheorie, Der ökonomische Mensch auf dem Weg durch die Sozialwissenschaften, Zeitschrift für philosophische Forschung 37 (1983), S. 256-274.

H.J. RAMSER (1987), Beschäftigung und Konjunktur, Springer, Berlin 1987.

D.A. ReDman (1993), Economics and the Philosophy of Science, Oxford University Press, New York/Oxford 1993.

W.H. RiKer und P.C. ORDESHOOK (1968), A Theory of the Calculus of Voting, American Political Science Review 62 (1968), S. 25-42.

J. Robinson (1972), The Second Crisis of Economic Theory, American Economic Review, Papers and Proceedings, 62.2 (1972), S. 1 - 10; deutsche Übersetzung: Die zweite Krise der ökonomischen Theorie, in: W. VogT (ed.), Seminar: Politische Ökonomie, Suhrkamp, Frankfurt 1973, S. $37-55$.

D. Romer (1993), The New Keynesian Synthesis, Journal of Economic Perspectives 7 (1993), Heft 1, S. $5-22$.

G. RonNING (1991), Mikroökonometrie, Springer, Berlin et al 1991.

SCHWEIZERISCHE AKADEMIE DER GEISTES- UND SOZIALWISSENSCHAFTEN [SAGW] (2010), Manifest zur Bedeutung, Qualitätsbeurteilung und Lehre der Methoden qualitativer Sozialforschung, SAGW Eigenverlag, Bern 2010.

P.A. SAMUelson und R.M. Solow (1960), Analytical Aspects of Anti-Inflation Policy, American Economic Review, Papers and Proceedings, 50.2 (1960), S. 177 - 194.

E. SCHLICHT (1977), Grundlagen der ökonomischen Analyse, Rowohlt, Hamburg 1977.

P.R. SENN (2006), The German Historical School in the History of Economic Thought, Journal of Economic Studies 32 (2006), S. 185 - 255.

J. SHEARMUR (1991), Popper Lakatos and Theoretical Progress in Economics, in: N. DE MARCHI und BLAUG (1991), S. 35 - 57.

H. SIEBERT (1998), Disziplinierung der nationalen Wirtschaftspolitik durch die internationale Kapitalmobilität, in: D. DUWENDAG (ed.), Finanzmärkte im Spannungsfeld von Globalisierung, Regulierung und Geldpolitik, Duncker und Humblot, Berlin 1998, S. 41 - 67. 
S.T. Silva (2009), On Evolutionary Technological Change and Economic Growth: Lakatos As a Starting Point for Appraisal, Journal of Evolutionary Economics 19 (2009), S. 111 - 135.

H.A. Simon (1955), A Behavioral Model of Rational Choice, Quarterly Journal of Economics 69 (1955), S. $99-118$.

A. SMITH (1776), An Inquiry into the Nature and Causes of the Wealth of Nations, London 1776; deutsche Übersetzung: Der Wohlstand der Nationen, Eine Untersuchung seiner Natur und seiner Ursachen, C.H. Beck, München 1974.

V.J. TARASCiO (1997), The Problem of Knowledge in Economics, Southern Economic Journal 64 (1997), S. $1-18$.

H. THEIL (1971), Principles of Econometrics, North-Holland, Amsterdam/London 1971.

W. Thomson (1999), The Young Person's Guide to Writing Economic Theory, Journal of Economic Literature 37 (1999), S. 157 - 183.

M. WeBER (1913), Über einige Kategorien der verstehenden Soziologie, reprinted in: M. WeBER, Gesammelte Aufsätze zur Wissenschaftslehre, Mohr (Siebeck), Tübingen, 7. Auflage 1988, S. 427 -474 .

J. WeIMANN (1984), Die Funktion der normalen Wissenschaft in den Wirtschaftswissenschaften, Zeitschrift für allgemeine Wissenschaftstheorie 15 (1984), S. 278 - 289.

M.R. West und L. Woessmann (2010), 'Every Catholic Child in a Catholic School': Historical Resistance to State Schooling, Contemporary Private Competition and Student Achievement across Countries, Economic Journal 120 (2010), S. F229 - F255.

M. Zavodny (2006), Does Watching Television Rot Your Mind? Estimates of the Effect on Test Scores, Economics of Education Review 25 (2006), S. 565 - 573. 